Publ. RIMS, Kyoto Univ.

14 (1978), $441-486$

\title{
Some Global Properties of Symmetric Diffusion Processes ${ }^{1}$
}

\author{
By \\ Kanji ICHIHARA*
}

Introduction

The principal purpose of this paper is to investigate some global properties of the diffusion process associated with a second order elliptic partial differential operator of self adjoint form. More specifically recurrence, transience and the rate of escape of the process will be discussed

Let $L$ be an elliptic operator on $R^{d}$ defined by

$$
L=\sum_{i, j=1}^{d} \frac{\partial}{\partial x_{i}}\left(a_{i j} \frac{\partial}{\partial x_{j}}\right)
$$

where $a_{i j}$ is smooth, symmetric and the coefficients' matrix $\left(a_{i j}(x)\right)_{1 \leqq i, j \leqq d}$ is strictly positive definite on $R^{d}$. We denote by $\left(X_{t}, P_{\tau}\right), x \in R^{d}$ the associated diffusion process.

Let $\varphi_{\rho}, \rho>1$ be the unique solution of the Dirichlet problem:

$$
L u=0 \quad \text { in } 1<|x|<\rho
$$

and

$$
u(x)=\left\{\begin{array}{lll}
1 & \text { on } & |x|=1 \\
0 & \text { on } & |x|=\rho .
\end{array}\right.
$$

The following fact is a special case of the celebrated Dirichlet principle :

" $\varphi_{\rho}$ is the unique solution of the problem minimizing an integral

Communicated by K. Itô, May 20, 1977.

* Department of Mathematics, Cornell University, Ithaca, New York, 14853, U.S. A. Present Address: Department of Applied Science, Kyushu University, Fukuoka 812 Japan

1) This paper is based on the Ph. D. Thesis, Cornell University, 1977. 


$$
\int_{1<|x|<p} \sum_{i, j=1}^{d} a_{i j} \frac{\partial u}{\partial x_{i}} \frac{\partial u}{\partial x_{j}} d x .
$$

over all $u \in H^{1,2}(\{x: 1<|x|<\rho\})$ with boundary values 1 and 0 on $|x|=1$ and $\rho$ respectively".

See Proposition 1.1 for the precise statement.

Hunt [10] made use of the above principle in his investigation on the existence of the 0 -order Green function of the process $\left(X_{t}, P_{x}\right)$.

We shall further develop this approach and adopt it in a study of the recurrent and transient properties. In Chapter 1 we shall treat the diffusion process associated with a strictly elliptic operator having measurable coefficients and Chapter 2 will be devoted to the study of a class of degenerate diffusion processes. The main parts of the proofs in these chapters will be performed in $L^{2}$-setting.

Our fundamental criterion for recurrence which will be proved in Chapters 1 and 2 is stated as follows :

"The process is recurrent if and only if

$$
\lim _{\rho \lambda+\infty} \int_{1<|x|<\rho} \sum_{i, j=1}^{d} a_{i j} \frac{\partial \varphi_{\rho}}{\partial x_{i}} \frac{\partial \varphi_{\rho}}{\partial x_{j}} d x=0 . "
$$

See Theorems 1.1 and 2. 1 for the details.

We denote by $A(x)$ the coefficients' matrix $\left(a_{i j}(x)\right)_{1 \leqq i, j \leqq d}$ and define

$$
\begin{aligned}
& E_{1}(x)=\frac{(A(x) x, x)}{|x|^{2}} \text { for } x \neq 0 \\
& E_{2}(x)=\frac{|x|^{2}}{\left(A^{-1}(x) x, x\right)} \text { for } x \neq 0
\end{aligned}
$$

and

$$
\bar{E}_{1}(r)=\int_{S^{d-1}} E_{1}(r \sigma) d \sigma \text { for } r>0
$$

where $A^{-1}(x)$ is the inverse of the matrix $A(x)$ and $d \sigma$ is the normalized uniform measure on $S^{d-1}$.

By means of the above criterion for recurrence, we shall prove 
in Chapter 1

Theorem A. If $\int_{1}^{\infty} r^{1-d} \bar{E}_{1}^{-1}(r) d r=+\infty$, then the process is recurrent.

Theorem B. If $\int_{1}^{\infty} r^{1-d} E_{2}^{-1}(r \sigma) d r<+\infty$ on a subset of $S^{d-1}$ with positive uniform measure, then the process is transient.

In Chapter 2 similar results will be obtained for the diffusion process governed by a hypoelliptic differential operator. See Theorems $\mathrm{C}$ and D.

It is to be mentioned that Brown [4] has proved similar results in case of $L=\sum_{i=1}^{d} \frac{\partial}{\partial x_{i}}\left(a(x) \frac{\partial}{\partial x_{i}}\right)$ where $a(x)$ is smooth, strictly positive on $R^{d}$, but the methods cannot be generalized to either of our cases.

In Chapter 3 we shall give some examples and compare our main results (Theorems $A-D$ ) with those which Friedman [7] and Hasminskii [8] have proved by the martingale method. It will then turn out that our test for recurrence contains that of Friedman and Hasminskii in the self adjoint case.

In the last chapter we shall give the exact escape rates to $\infty$ for the diffusion paths governed by a uniformly elliptic operator; this is an extension of Dvoretsky and Erdös' test [6] for the Brownian motion in space.

The author would like to express sincere thanks to Professor Harry Kesten for helpful advice and all his inspiration. Gratitude is also given to Professors Kiyosi Itô, Hiroshi Kunita and S. R. S. Varadhan for valuable suggestions and helpful criticism.

\section{Notations and Definitions}

Let $\Omega$ be an open domain $R^{d}$.

1. $C(\bar{\Omega})$ : The class of real continuous functions in $\bar{\Omega}$.

2. $C^{1}(\bar{\Omega})$ : The subclass of $C(\bar{\Omega})$ functions having continuous first partial derivatives on $\Omega$ which can be extended continuously to $\bar{\Omega}$.

3. $C_{0}^{\infty}(\Omega)$ : The class of real, infinitely differentiable functions with compact support in $\Omega$.

4. $H^{1,2}(\Omega)$ : The completion of $C^{1}(\bar{\Omega})$ with respect to the norm 


$$
\|u\|_{H^{1,2(\Omega)}}=\sqrt{\int_{\Omega}\left\{u^{2}+\sum_{i=1}^{d}\left(\frac{\partial u}{\partial x_{i}}\right)^{2}\right\} d x} .
$$

5. $H_{l \circ}^{1.2}{ }_{c}(\Omega)$ : The class of real measurable functions which are of the $H^{1,2}\left(\Omega^{\prime}\right)$ class for every bounded open $\Omega^{\prime}$ with closure in $\Omega$.

6. $H_{0}^{1,2}(\Omega)$ : The closure of $C_{0}^{\infty}(\Omega)$ in $H^{1,2}(\Omega)$.

7. Let the matrix $A(x)=\left(a_{i j}(x)\right)_{1 \leqq i, j \leqq d}$ be symmetric, positive definite, with real components $a_{i j}(x), 1 \leqq i, j \leqq d$ locally bounded, measurable in $R^{d}$. Let the function $k(x)$ be strictly positive, continuous and integrable on $\Omega$. Define a quadratic form by

$$
D(u ; \Omega)=\int_{\Omega} \sum_{i=1}^{d} a_{i j} \frac{\partial u}{\partial x_{i}} \frac{\partial u}{\partial x_{j}} d x .
$$

8. $H_{0}^{1,2}(\Omega: k d x)$ : The completion of $C_{0}^{\infty}(\Omega)$ with respect to the norm

$$
\|u\|_{H_{0}^{1,2(\Omega: k d x)}}=\sqrt{\int_{\Omega} u^{2} k d x+D(u ; \Omega) .}
$$

\section{Chapter 1. Criteria for Recurrence and Transience $\mathbb{I}$. Strictly elliptic differential operator}

Suppose we are given an elliptic operator defined on $R^{d}$ as

$$
L=\sum_{i, j=1}^{d} \frac{\partial}{\partial x_{i}}\left(a_{i j} \frac{\partial}{\partial x_{j}}\right)
$$

where $a_{i j}$ is locally bounded, measurable, symmetric and $L$ is locally uniformly elliptic, i. e., for each compact subset $K \subset R^{d}$, there exists a constant $\lambda=\lambda(K)>1$ such that

$$
\lambda^{-1}|\xi|^{2} \leqq \sum_{i, j=1}^{d} a_{i j}(x) \xi_{i} \xi_{j} \leqq \lambda|\xi|^{2}
$$

in $K$ and for all $\xi \in R^{d}$.

Let $D$ be a bounded domain in $R^{d}$. A function $u$ on $D$ is called a weak solution of $L u=f$ for a given $f \in L^{2}(D)$ if it has weak derivatives $\frac{\partial u}{\partial x_{i}}, i=1, \ldots, d$ which belong to $L_{l o c}^{2}(D)$ and

$$
\int_{D} \sum_{i, j=1}^{d} a_{i j} \frac{\partial v}{\partial x_{i}} \frac{\partial u}{\partial x_{j}} d x=-\int_{D} v \cdot f d x
$$

is fulfilled for all $v \in C_{0}^{\infty}(D)$.

Let $R_{\alpha}, \alpha>0$ be a family of linear transformations from $L^{\infty}(D)$ to $C(D)$, the set of all continuous functions on $D$, satisfying the resolvent equation

$$
R_{\alpha}-R_{\beta}+(\alpha-\beta) R_{\alpha} R_{\beta}=0 \text { for all } \alpha, \beta>0
$$


and the sub Markov property: $0 \leqq \alpha R_{\alpha} f \leqq 1$ if $0 \leqq f \leqq 1$. If $u=R_{\alpha} f$ satisfies

$$
(\alpha-L) u \stackrel{\text { W }}{=} f \text { for all } f \in L^{\infty}(D),
$$

$R_{\alpha}$ is called an $L$-diffusion resolvent. It is easy to see from (1.4) that $R_{\alpha}$ is a one to one linear operator from $L^{\infty}(D)$ to $C(D)$. Set $D(A)=\left\{R_{\alpha} f: f \in L^{\infty}(D)\right\}$, independent of $\alpha>0$ by (1.3) and define for $u \in D(A), A u=\left(\alpha-R_{\alpha}^{-1}\right) u(=L u) . A$ with the domain $D(A)$ is called the generator of the resolvent.

The existence of the unique minimal diffusion process associated with the operator $L$ in an arbitrary bounded domain has been proved by Kanda [12] and Kunita [14]. Their construction is based on the Green function constructed by Littman, Stampacchia and Weinberger [16] and Stampacchia [19] respectively. Note that Kunita treats the more general case $L=\sum_{i, y=1}^{d} \frac{\partial}{\partial x_{i}}\left(a_{i j} \frac{\partial}{\partial x_{j}}\right)+\sum_{i=1}^{d} b_{i} \frac{\partial}{\partial x_{i}}$.

We shall explain briefly their process. Let $D$ be as above. Littman et al. [16] and Stampacchia [19] have shown that there exists a unique Green operator $G_{\alpha}, \alpha \geqq 0$ such that $G_{\alpha}$ transforms $L^{\infty}(D)$ into $C(D)$ and satisfies (1.3) and (1.4). In particular, if $\partial D$ is regular ( $\partial D$ is of the $C^{1}$-class, for example). $\left(G_{\alpha} f\right)(x)$ converges to 0 as $x \in D$ approaches a boundary point.

Making use of the above Green operator $G_{\alpha}$, Kunita and Kanda have constructed a unique minimal diffusion process $\left(X_{t}^{D}, \zeta^{D}, P_{x}^{D}\right)$, $x \in D$ for which

$$
\text { (1. 5) } G_{\alpha} f(x)=E_{x}\left[\int_{0}^{\zeta^{D}} e^{-\alpha t} f\left(X_{t}^{D}\right) d t\right] \text {, for all } f \in L^{\infty}(D) \text { and } \alpha \geqq 0
$$

where $\zeta^{D}$ is the killing time for the process.

It is to be noted that the process has the communication property i. e., for each open subset $U$ of $D$ and each $x \in D, \quad P_{x}\left[x_{t}^{D} \in U\right.$ for some $\left.t \in\left(0, \zeta^{D}\right)\right]>0$, since the 0 -order Green function is strictly positive. See Littman et al. [16], section 7. It is also easy to see from (1.5) with $\alpha=0$ and $f \equiv 1$ that the minimal diffusion is transient.

Combining the local existence by Kanda and Kunita with Courrege and Priouret [5], we will now construct a minimal diffusion process 
in $R^{d}$. Let $\Sigma_{\rho}$ be the open sphere with radius $\rho$ and the origin as a center. We then have a class of local diffusions $\left(X_{t}^{\eta_{n}}, \zeta^{\Sigma_{n}}\right.$, $\left.P_{x}^{\Sigma_{n}}\right), x \in \Sigma_{n}, n \geqq 1$. Proposition 2.4 of Kunita [14] asserts that the stopped process $\left(X_{t}^{S_{n+1}}, \sigma_{n}^{n+1}, P_{x}^{\Sigma_{n+1}}\right), x \in \Sigma_{n}$ where $\sigma_{n}^{n+1}=\inf \{t \geqq$ $\left.0:\left|x_{t}^{\Sigma_{n+1}}\right| \geqq n\right\}$ is equivalent in law to the process $\left(X_{t}^{\Sigma_{n}}, \zeta^{\Sigma_{n}}, P_{x}^{\Sigma_{n}}\right), x \in$ $\Sigma_{n}$. Consequently by Theorem 2 of Courrege and Priouret [5], we see that there exists a unique standard diffusion process $X_{t}$ in $R^{d}$ which is locally equivalent to that of Kanda and Kunita. Let $\sigma_{\rho}=$ $\inf \left\{t \geqq 0:\left|x_{t}\right| \geqq \rho\right\}$. Note that $\sigma_{\rho}<+\infty$ a. e. since $\left(X_{t}^{\Sigma_{\rho}+1}, \zeta^{\Sigma_{\rho}+1}, P_{x}^{\Sigma_{\rho}+1}\right)$, $x \in \Sigma_{\rho+1}$ is transient. See the above remark. Now define $\zeta=\lim _{\rho+\infty} \sigma_{\rho}$. Then the process $\left(X_{t}, \zeta, P_{x}\right), x \in R^{d}$ is a desired minimal diffusion process in $R^{d}$.

What we are concerned with in this chapter is to investigate the recurrent and transient properties of the process $\left(X_{t}, P_{x}\right)$ constructed above.

Definition 1. 1. The process will be said to be recurrent if $P_{x}\left[X_{t}\right.$ $\in U$ for some $t>0] \equiv 1$ for any open subset $U$ of $R^{d}$, otherwise it will be called transient.

Remark 1. 1. From the definition, it is obvious that $\zeta=+\infty$ a. e. if the process is recurrent, and transience is equivalent to

$$
P_{x}\left[\lim _{t / 5}\left|x_{t}\right|=+\infty\right] \equiv 1 .
$$

(1.6) will be proved in Corollary 1.2 after some preparation.

Our main results in this chapter are Theorems A and B. In order to prove them, we introduce the stopping time

$$
\tau_{1}=\inf \left\{t \geqq 0:\left|x_{t}\right| \leqq 1\right\}
$$

and let $\sigma_{\rho}, \rho>1$ be as before. Define

$$
\begin{array}{ll}
\varphi_{\rho}(x)=P_{x}\left[\tau_{1}<\sigma_{\rho}\right], & x \in R^{d} \\
\varphi_{\infty}(x)=P_{x}\left[\tau_{1}<\zeta\right], & x \in R^{d} .
\end{array}
$$

From the definition of $\zeta$, it is obvious that 


$$
\lim _{\rho>+\infty} \varphi_{\rho}(x)=\varphi_{\infty}(x), \quad x \in R^{d} .
$$

The following proposition is essentially due to Littman, Stampacchia and Weinberger [16].

Proposition 1. 1. For $1<\rho<+\infty$, (i) $\varphi_{\rho} \in H_{0}^{1,2}\left(\Sigma_{\rho}\right)$ and is a weak solution of $L u=0$ in $\Sigma_{\rho}-\bar{\Sigma}_{1}$, and converges to the boundary values 1 and 0 as $|x| \searrow 1$ and $|x| \nearrow \rho$ respectively.

(ii) $\varphi_{\rho}$ is Hölder continuous in $\Sigma_{\rho}-\bar{\Sigma}_{1}$, more precisely, for any compact subset $K \subset \Sigma_{\rho_{0}}-\bar{\Sigma}_{1}, \rho_{0}>1$, there exist positive constants $C=$ $C\left(K, \rho_{0}\right)$ and $\alpha=\alpha\left(K, \rho_{0}\right)$ such that

$$
\left|\varphi_{\rho}(x)-\varphi_{\rho}\left(x^{\prime}\right)\right| \leqq C|| \varphi_{\rho} \|_{L^{2}\left(\Sigma_{\rho_{0}}-\Sigma_{1}\right)} \cdot\left|x-x^{\prime}\right|^{\alpha}
$$

for every $x, x^{\prime} \in K, \rho \in\left[\rho_{0},+\infty\right)$.

(iii) $\varphi_{\rho}-\phi_{\rho} \in H_{0}^{1,2}\left(\Sigma_{\rho}-\bar{\Sigma}_{1}\right)$ and $\varphi_{\rho}$ minimizes an integral

$$
\int_{1<|x|<p} \sum_{i, j=1}^{d} a_{i j} \frac{\partial u}{\partial x_{i}} \frac{\partial u}{\partial x_{j}} d x
$$

over all $u \in H_{0}^{1,2}\left(\Sigma_{\rho}-\bar{\Sigma}_{1}\right)+\left\{\phi_{\rho}\right\}$, where $\phi_{\rho}$ is any fixed function $C_{0}^{\infty}\left(\Sigma_{\rho}\right)$ such that $\phi_{\rho}(x) \equiv 1$ on $\bar{\Sigma}_{1}$ and $H_{0}^{1,2}\left(\Sigma_{\rho}-\bar{\Sigma}_{1}\right)+\left\{\phi_{\rho}\right\}=\left\{\phi+\phi_{\rho}: \phi \in\right.$ $\left.H_{0}^{1,2}\left(\Sigma_{\rho}-\bar{\Sigma}_{1}\right)\right\}$.

Remark 1. 2. $H_{0}^{1,2}\left(\Sigma_{\rho}-\bar{\Sigma}_{1}\right)+\left\{\phi_{\rho}\right\}$ is independent of the choice of $\phi_{\rho}$ as long as $\phi_{\rho}$ satisfies the above conditions. It is also to be noted that $H_{0}^{1,2}\left(\Sigma_{\rho}-\bar{\Sigma}_{1}\right)+\left\{\phi_{\rho}\right\}$ is increasing as $\rho \nearrow+\infty$ after the trivial extension of elemets to $\Sigma_{\rho}^{c}$.

Proof of Proposition 1. 1. We first show that there exists a function on $\Sigma_{\rho}$ which satisfies all the above properties and is identically equal to 1 on $\bar{\Sigma}_{1}$.

Combining Theorems 2.1 and 2.3 of Littman et al. [16], we see that there exists a unique function $u_{\rho}$ on $\Sigma_{\rho}-\bar{\Sigma}_{1}$ which is a weak solution of $L u=0$ in $\Sigma_{\rho}-\bar{\Sigma}_{1}$, Hölder continuous i. e. (1. 7) holds with $u_{\rho}$ in place of $\varphi_{\rho}$, and $u_{\rho}-\varphi_{\rho} \in H_{0}^{1,2}\left(\Sigma_{\rho}-\bar{\Sigma}_{1}\right)$. We will prove the second property of (iii) for $u_{\rho}$. Since $u_{\rho}$ is a weak solution in $\Sigma_{\rho}-\bar{\Sigma}_{1}$ and $L$ is uniformly elliptic on $\bar{\Sigma}_{\rho}-\bar{\Sigma}_{1}$, a simple limiting procedure 
gives that

$$
\int_{\Sigma_{\rho}-\Sigma_{1}} \sum_{i, j=1}^{d} a_{i j} \frac{\partial u_{\rho}}{\partial x_{i}} \frac{\partial \psi}{\partial x_{j}} d x=0 \text { for all } \phi \in H_{0}^{1,2}\left(\Sigma_{\rho}-\bar{\Sigma}_{1}\right) \text {. }
$$

Suppose $\varphi \in H_{0}^{1,2}\left(\Sigma_{\rho}-\bar{\Sigma}_{1}\right)+\left\{\phi_{\rho}\right\}$ and compute

$$
\begin{aligned}
& \int_{\Sigma_{\rho}-\Sigma_{1}} \sum_{i, j=1}^{d} a_{i j} \frac{\partial \varphi}{\partial x_{i}} \frac{\partial \varphi}{\partial x_{j}} d x \\
& =\int_{\Sigma_{\rho}-\Sigma_{1}} \sum_{i, j=1}^{d} a_{i j} \frac{\partial\left\{u_{\rho}+\left(\varphi-u_{\rho}\right)\right\}}{\partial x_{i}} \frac{\partial\left\{u_{\rho}+\left(\varphi-u_{\rho}\right)\right\}}{\partial x_{j}} d x \\
& =\int_{\Sigma_{\rho}-\Sigma_{1}} \sum_{i, j=1}^{d} a_{i j} \frac{\partial u_{\rho}}{\partial x_{i}} \frac{\partial u_{\rho}}{\partial x_{j}}+2 \int_{\Sigma_{\rho}-\Sigma_{1}} \sum_{i, j=1}^{d} a_{i j} \frac{\partial u_{\rho}}{\partial x_{i}} \frac{\partial\left\{\varphi-u_{\rho}\right\}}{\partial x_{j}} d x \\
& +\int_{\Sigma_{\rho}-\Sigma_{1}} \sum_{i, j=1}^{d} a_{i j} \frac{\partial\left\{\varphi-u_{\rho}\right\}}{\partial x_{i}} \frac{\partial\left\{\varphi-u_{\rho}\right\}}{\partial x_{j}} d x .
\end{aligned}
$$

Since $\varphi-u_{\rho} \in H_{0}^{1,2}\left(\Sigma_{\rho}-\bar{\Sigma}_{1}\right)$, the second term is equal to zero as we saw above. Hence we obtain

$$
\int_{\Sigma_{\rho}-\Sigma_{1}} \sum_{i, j=1}^{d} a_{i j} \frac{\partial \varphi}{\partial x_{i}} \frac{\partial \varphi}{\partial x_{j}} d x \geqq \int_{\Sigma_{\rho}-\Sigma_{1}} \sum_{i, j=1}^{d} a_{i j} \frac{\partial u_{\rho}}{\partial x_{i}} \frac{\partial u_{\rho}}{\partial x_{j}} d x .
$$

This shows that $u_{\rho}$ minimizes (1.8).

By Corollary 9. 1 of Littman et al. [16], all the boundary points of $\Sigma_{\rho}-\bar{\Sigma}_{1}$ are regular. This combined with Theorem 3.1 and Definition 3. 1 of Littman et al. [16] implies that $u_{\rho}$ has the boundary values 1 and 0 on $|x|=1$ and $\rho$ respectively.

Now let us extend the function $u_{\rho}$ by

$$
\tilde{u}_{\rho}(x)= \begin{cases}u_{\rho}(x) & \text { on } \Sigma_{\rho}-\bar{\Sigma}_{1} \\ 1 & \text { on } \bar{\Sigma}_{1} .\end{cases}
$$

We want to prove that $\tilde{u}_{\rho} \in H_{0}^{1,2}\left(\Sigma_{\rho}\right)$. From the fact that $u_{\rho}-\phi_{\rho} \in$ $H_{0}^{1,2}\left(\Sigma_{\rho}-\bar{\Sigma}_{1}\right)$, we can choose a sequence $w_{n}, n \geqq 1 \in C_{0}^{\infty}\left(R^{d}\right)$ with support in $\Sigma_{\rho}-\bar{\Sigma}_{1}$ such that

$$
\lim _{n>+\infty}\left\|w_{n}-\left(u_{\rho}-\phi_{\rho}\right)\right\|_{H_{0}^{1,2}\left(\Sigma_{p}-\Sigma_{1}\right)}=0
$$

Note that a sequence $w_{n}+\phi_{\rho}, n \geqq 1 \in H_{0}^{1,2}\left(\Sigma_{\rho}\right)$ and is Cauchy in this space, hence there exists a unique element of $H_{0}^{1,2}\left(\Sigma_{\rho}\right)$ to which 
$w_{n}+\phi_{\rho}$ converges to $H_{0}^{1,2}\left(\Sigma_{\rho}\right)$. It is also possible to choose a subsequence of $w_{n}+\phi_{\rho}$ so that it converges to $\tilde{u}_{\rho}$ a. e. on $\Sigma_{\rho}$. Combining all of this, we see that $\tilde{u}_{\rho} \in H_{0}^{1,2}\left(\Sigma_{\rho}\right)$. Thus we have constructed a desired function $\tilde{u}_{\rho}$.

We now proceed with the proof of Proposition 1.1. It suffices to verify that

$$
\varphi_{\rho}(x)=\tilde{u}_{\rho}(x) \text { for all } x \in \Sigma_{\rho}-\bar{\Sigma}_{1} \text {. }
$$

Since $\tilde{u}_{\rho}$ is a weak solution of $L u=0$ in $\Sigma_{\rho}-\bar{\Sigma}_{1}$, it follows from Theorem 2. 1 of Kunita [14] that $\tilde{u}_{\rho}$ is 0 -harmonic i. e.

$$
E_{x}\left[\tilde{u}_{\rho}\left(x_{\tau_{v c}}\right)\right]=\tilde{u}_{\rho}(x), \quad x \in V
$$

for all sets $V$ such that $\bar{V} \subset \Sigma_{\rho}-\bar{\Sigma}_{1}$, where $\tau_{v^{c}}$ is the hitting time for the set $V^{c}$. Now letting $V \nearrow \Sigma_{\rho}-\bar{\Sigma}_{1}$, we obtain by the continuity of $\tilde{u}_{\rho}$ and the fact $P_{x}\left[\tau_{1}=\sigma_{\rho}=+\infty\right]=0$ for every $x \in \Sigma_{\rho}-\bar{\Sigma}_{1}$, see the remark on page 446 , that

$$
\varphi_{\rho}(x)=\tilde{u}_{\rho}(x) \text { for all } x \in \Sigma_{\rho}-\bar{\Sigma}_{1} .
$$

This completes the proof.

The next proposition is a major step in our proof.

Proposition 1. 2. $\varphi_{\infty}$ satisfies (1.7) with $\rho=+\infty$ in the inequality, $\varphi_{\infty} \in H_{l o c}^{1,2}\left(R^{d}\right)$ and

$$
\lim _{\rho>+\infty} D\left(\varphi_{\rho}: \Sigma_{\rho}\right)=D\left(\varphi_{\infty}: R^{d}\right) .
$$

For the proof of this proposition, we shall make use of the Hilbert space $H_{0}^{1,2}\left(R^{d}: k d x\right)$, where $k(x)$ is strictly positive, continuous and integrable on $R^{d}$. Before proceeding to the proof, we note that if $u$ is an element of $H_{0}^{1,2}\left(R^{d}: k d x\right)$, then (i) $u$ and $\frac{\partial u}{\partial x_{i}} \in L_{l o c}^{2}\left(R^{d}\right)$, $i=1, \ldots, d$.

(ii) $\|u\|_{H_{0}^{1,2}\left(R^{d}: k d x\right)}=\sqrt{\int_{R^{d}}\left\{u^{2} k+\sum_{i, j=1}^{d} a_{i j}-\frac{\partial u}{\partial x_{i}} \frac{\partial u}{\partial x_{j}}\right\} d x}$.

Proof of Proposition 1.2. The first statement is immediate from 
Proposition 1.1 (ii) and the pointwise convergence of $\varphi_{\rho}$ to $\varphi_{\infty}$.

By Proposition 1.1 (iii) and Remark 1.2, we have that

$$
D\left(\varphi_{\rho}: \Sigma_{\rho}\right)=\int_{\Sigma_{\rho}-\Sigma_{1}} \sum_{i, j=1}^{d} a_{i j} \frac{\partial \varphi_{\rho}}{\partial x_{i}} \frac{\partial \varphi_{\rho}}{\partial x_{j}} d x
$$

is non increasing as $\rho \nearrow+\infty$. Therefore the limit of $D\left(\varphi_{\rho}: \Sigma_{\rho}\right)$ exists and is non negative.

Using the above fact, we first show that

$$
\lim _{\rho, \rho^{\prime}>+\infty} D\left(\varphi_{\rho}-\varphi_{\rho^{\prime}}: R^{d}\right)=0 .
$$

Without loss of generality, we can assume that $\rho<\rho^{\prime}$. Then

$$
\begin{aligned}
D & \left(\varphi_{\rho}-\varphi_{\rho^{\prime}}: R^{d}\right)=\int_{\Sigma_{\rho^{\prime}}-\Sigma_{1}} \sum_{i, j=1}^{d} a_{i j} \frac{\partial\left\{\varphi_{\rho}-\varphi_{\rho^{\prime}}\right\}}{\partial x_{i}} \frac{\partial\left\{\varphi_{\rho}-\varphi_{\rho^{\prime}}\right\}}{\partial x_{j}} d x \\
& =\int_{\Sigma_{\sigma}-\Sigma_{1}} \sum_{i, j=1}^{d} a_{i j} \frac{\partial \varphi_{\rho}}{\partial x_{i}} \frac{\partial \varphi_{\rho}}{\partial x_{j}} d x-2 \int_{\Sigma_{\rho^{\prime}-\Sigma_{1}}} \sum_{i, j=1}^{d} a_{i j} \frac{\partial \varphi_{\rho}}{\partial x_{i}} \frac{\partial \varphi_{\rho^{\prime}}}{\partial x_{j}} d x \\
& +\int_{\Sigma_{\rho^{\prime}-\Sigma_{1}}} \sum_{i, j=1}^{d} a_{i j} \frac{\partial \varphi_{\rho^{\prime}}}{\partial x_{i}} \frac{\partial \varphi_{\rho^{\prime}}}{\partial x_{j}} d x .
\end{aligned}
$$

On the other hand, the property (iii) of Proposition 1.1 implies that

$$
\int_{\Sigma_{\rho^{\prime}}-\Sigma_{1}} \sum_{i, j=1}^{d} a_{i j} \frac{\partial \varphi_{\rho^{\prime}}}{\partial x_{i}} \frac{\partial\left\{\varphi_{\rho^{\prime}}-\varphi_{\rho}\right\}}{\partial x_{j}} d x=0
$$

since $\varphi_{\rho}-\varphi_{\rho^{\prime}} \in H_{0}^{1,2}\left(\Sigma_{\rho^{\prime}}-\bar{\Sigma}_{1}\right)$. Therefore one has that

$$
\begin{aligned}
D\left(\varphi_{\rho}-\varphi_{\rho^{\prime}}: R^{d}\right) & =\int_{\Sigma_{\rho}-\Sigma_{1}} \sum_{i, j=1}^{d} a_{i j} \frac{\partial \varphi_{\rho}}{\partial x_{i}} \frac{\partial \varphi_{\rho}}{\partial x_{j}} d x \\
& -\int_{\Sigma_{\rho^{\prime}-\Sigma_{1}}} \sum_{i, j=1}^{d} a_{i j} \frac{\partial \varphi_{\rho^{\prime}}}{\partial x_{i}} \frac{\partial \varphi_{\rho^{\prime}}}{\partial x_{j}} d x \\
& =D\left(\varphi_{\rho}: \Sigma_{\rho}\right)-D\left(\varphi_{\rho^{\prime}}: \Sigma_{\rho^{\prime}}\right)
\end{aligned}
$$

(1.11) now follows from the existence of $\lim _{\rho>+\infty} D\left(\varphi_{\rho}: \Sigma_{\rho}\right)$.

It is now easy to see that

$$
\varphi_{\rho} \in H_{0}^{1,2}\left(R^{d}: k d x\right)
$$

and

$$
\lim _{\rho, \rho^{\prime} \lambda+\infty}\left\|\varphi_{\rho}-\varphi_{\rho^{\prime}}\right\|_{H_{0}^{1,2}\left(R^{d: k d x)}\right.}=0
$$


since $\varphi_{\rho}(x) \nearrow \varphi_{\infty}(x) \leqq 1$ for all $x \in R^{d}$ as $\rho \nearrow+\infty$. By the completeness of the space $H_{0}^{1,2}\left(R^{d}: k d x\right)$, there exists a unique element $\tilde{\varphi} \in H_{0}^{1,2}\left(R^{d}\right.$ : $k d x)$ such that

$$
\lim _{\rho \rho^{\top}+\infty}\left\|\varphi_{\rho}-\tilde{\varphi}\right\|_{H_{0}^{1,2}\left(R^{d}: k d z\right)}=0
$$

Since $\varphi_{\rho}$ converges to $\varphi_{\infty}$ pointwise in $R^{d}, \tilde{\varphi}$ has to be $\varphi_{\infty}$. Thus we have shown that

$$
\lim _{\rho \nearrow+\infty}\left\|\varphi_{\rho}-\varphi_{\infty}\right\|_{H_{0}^{1,2}\left(R^{d}: k d x\right)}=0
$$

which gives $\varphi_{\infty} \in H_{0}^{1,2}\left(R^{d}: k d x\right)$ and

$$
\lim _{\rho>+\infty} D\left(\varphi_{\rho}: \Sigma_{\rho}\right)=D\left(\varphi_{\infty}: R^{d}\right) . \quad \text { Q. E. D. }
$$

Combining Propositions 1.1 and 1.2, we obtain

Corollary 1.1. $\varphi_{\infty}$ is a weak solution of $L u=0$ in the exterior domain $R^{d}-\bar{\Sigma}_{1}$, which has the boundary value 1 on $|x|=1$ and is strictly positive.

Proof. From Proposition 1.1 (i), we have

$$
\int_{\Sigma_{\rho}-\Sigma_{1}} \sum_{i, j=1}^{d} a_{i j} \frac{\partial \varphi_{\rho}}{\partial x_{i}} \frac{\partial \psi}{\partial x_{j}} d x=0 \text { for } \phi \in C_{0}^{\infty}\left(\Sigma_{\rho}-\bar{\Sigma}_{1}\right) .
$$

Since we can replace the domain $\Sigma_{p}-\overline{\Sigma_{1}}$ in the left hand side of the above equality by $R^{d}-\bar{\Sigma}_{1}$, we obtain from Proposition 1.2

$$
\int_{R_{d}-\Sigma_{1}} \sum_{i, j=1}^{d} a_{i j} \frac{\partial \varphi_{\infty}}{\partial x_{i}} \frac{\partial \psi}{\partial x_{j}} d x=0 \text { for every } \phi \in C_{0}^{\infty}\left(R^{d}-\bar{\Sigma}_{1}\right) .
$$

This implies that $\varphi_{\infty}$ is a weak solution of $L u=0$ in $R^{d}-\bar{\Sigma}_{1}$.

The boundary condition on $|x|=1$ follows from the fact $\varphi_{\rho}(x) \nearrow$ $\varphi_{\infty}(x)$ as $\rho \nearrow+\infty$ for all $x \in R^{d}$, and Proposition 1.1 (i).

Since the killed process $\left(X_{t}, \sigma_{\rho}, P_{x}\right), x \in \Sigma_{\rho}$ has the communication property i. e.

$$
P_{x}\left[x_{t} \in U \text { for some } t \in\left(0, \sigma_{\rho}\right)\right]>0
$$

for every open subset $U$ of $\Sigma_{\rho}$ and every $x \in \Sigma_{\rho}$, it is obvious that 


$$
P_{x}\left[\tau_{1}<\sigma_{\rho}\right]>0 \text { for } x \in \Sigma_{\rho}-\bar{\Sigma}_{1} .
$$

Since $\varphi_{\infty}(x) \geqq \varphi_{\rho}(x), x \in R^{d}$, it follows that $\varphi_{\infty}(x)>0, x \in R^{d}$.

Q. E. D.

It should be noted that in the above results the unit sphere centered at the origin can be replaced by an arbitrary sphere in $R^{d}$.

From the corollary and the remark above, we have the following

Lemma 1. 1. $\left(X_{t}, P_{x}\right)$ is recurrent if and only if $\varphi_{\infty} \equiv 1$ on $R^{d}$.

Proof. It suffices to show that if $\varphi_{\infty} \equiv 1$, then

$$
P_{x}\left[x_{t} \in \bar{U} \text { for some } t>0\right] \equiv 1
$$

for any ball $U$ in $R^{d}$ such that $\partial U \cap \partial \Sigma_{1}=\phi$.

Let $\mathscr{F}_{t}$ be the the $\sigma$-field generated by $X_{s}, s \leqq t$. Define

$$
Y_{t} \equiv \begin{cases}P_{x_{t}}\left[\tau_{0}<\zeta\right] & \text { if } t<\tau_{0} \\ 1 & \text { otherwise, }\end{cases}
$$

where $\tau_{0}=\inf \left\{t \geqq 0: x_{t} \in \bar{U}\right\}$. Then it is easy to see that

$$
Y_{t}=P\left[\tau_{0}<\zeta \mid \mathscr{F}_{t}\right]
$$

and so $Y_{t}$ is a martingale. From the martingale convergence theorem, one has

$$
\lim _{t>+\infty} Y_{t}=\left\{\begin{array}{lll}
1 & \text { if } & \tau_{0}<+\infty \\
0 & \text { if } & \tau_{0}=+\infty
\end{array} \quad\right. \text { a. e. }
$$

On the other hand, from the continuity of $\varphi_{U}(x)=P_{x}\left[\tau_{0}<\zeta\right]$,

$$
\lim _{t>+\infty} Y_{t} \geqq \lim _{t+\infty} P_{x_{t}}\left[\tau_{U}<\zeta\right] \geqq \inf _{|x|=1} \varphi_{U}(x)>0
$$

since each sample path $x_{t}$ hits the unit sphere infinitely often with probability 1 . This implies that $\tau_{0}$ must be finite with probability 1 .

Q. E. D.

Corollary 1.2. If the process is transient, then $\varphi_{\infty}$ cannot attain the maximum value 1 in the exterior domain $R^{d}-\bar{\Sigma}_{1}$ and (1.6) holds. 
Proof. Suppose $\varphi_{\infty}$ attains the value 1 at $x_{0} \in R^{d}-\bar{\Sigma}_{1}$. Let $V$ be an open bounded domain such that $x_{0} \in V$ and $\bar{V} \subset R^{d}-\bar{\Sigma}_{1}$. Define $\tau_{v^{c}}$ as before. Then it is easy to see from the strong Markov property that

$$
\varphi_{\infty}\left(x_{0}\right)=E_{x_{0}}\left[\varphi_{\infty}\left(x_{t \wedge \tau}\right)\right]
$$

Combining this equality with the communication property of the process $x_{t}, t<\tau_{V^{c}}$ and the continuity of $\varphi_{\infty}$, we obtain

$$
\varphi_{\infty}(x)=1 \text { on } \bar{V} \text {. }
$$

Since $V$ is arbitrary, we have $\varphi_{\infty} \equiv 1$ on $R^{d}-\bar{\Sigma}_{1}$. However this cannot happen by Lemma 1.1. This proves the first assertion.

To prove (1.6), we first note that there exist a point $x_{1} \in R^{d}$ and a compact subset $K$ of $R^{d}$ such that

$$
P_{x_{1}}\left[x_{t} \text { hits } K \text { i.o. as } t \nearrow+\infty\right]>0
$$

if (1.6) is false. We can assume $K=\bar{\Sigma}_{1}$ without loss of generality. Define a sequence of stopping times for a fixed $\rho>1$,

$$
\begin{aligned}
& \tau_{1}^{1}=\tau_{1} \\
& \sigma_{\rho}^{1}=\inf \left\{t \geqq \tau_{1}:\left|x_{t}\right| \geqq \rho\right\} \\
& \tau_{1}^{n}=\inf \left\{t \geqq \sigma_{\rho}^{n-1}:\left|x_{t}\right| \leqq 1\right\} \\
& \sigma_{p}^{n}=\inf \left\{t \geqq \tau_{1}^{n}:\left|x_{t}\right| \geqq \rho\right\} \quad \text { for } n \geqq 2 .
\end{aligned}
$$

Note that $\sigma_{\rho}^{n}$ is finite with probability 1 for al $n$. Observe that

$$
\left\{x_{t} \text { hits } K \text { i.o. as } t \nearrow+\infty\right\} \subseteq\left\{\tau_{1}^{n}<\zeta\right\}
$$

for every positive integer $n$. We want to estimate

$$
P_{x_{1}}\left[\tau_{1}^{n}<\zeta\right]=P_{x_{1}}\left[\tau_{1}^{1}<\sigma_{\rho}^{1}<\tau_{1}^{2}<\cdots<\sigma_{\rho}^{n-1}<\tau_{1}^{n}<\zeta\right] .
$$

By repeated use of the strong Markov property, the right hand side is equal to

$$
\begin{aligned}
& E_{x_{1}}\left[\tau_{1}^{1}<+\infty, E_{\tau_{1}^{1}}\left[\tau_{1}^{2}<+\infty, E_{x_{\tau 2}}\left[\ldots \left[\tau_{1}^{n-1}<+\infty\right.\right. \text {, }\right.\right. \\
& E_{\tau_{1}^{n-1}}\left[P_{\substack{x \\
\sigma_{o}^{n-1}}}\left[\tau_{1}^{n}<\zeta\right] \ldots\right] .
\end{aligned}
$$

Set $c=\sup _{|x|=\rho} \varphi_{\infty}(x)$, then $c$ is strictly less than 1 by the first assertion. From (1.15) and (1.16), we obtain 


$$
P_{x_{1}}\left[\tau_{1}^{n}<\zeta\right] \leqq c^{n-1} P_{x_{1}}\left[\tau_{1}^{1}<\zeta\right] \searrow 0
$$

as $n \nearrow+\infty$.

Hence $P_{x_{1}}\left[x_{t}\right.$ hits $K$ i.o. as $\left.t \nearrow+\infty\right]=0$. Thus we have $\operatorname{shown}(1.6)$.

Q. E. D.

We now prove the following fundamental criterion for recurrence.

Theorem 1.1. $\left(X_{t}, P_{x}\right)$ is recurrent if and only if

$$
\lim _{\rho \lambda+\infty} D\left(\varphi_{\rho}: \Sigma_{\rho}\right)=0 \text {. }
$$

Proof. By virtue of Proposition 1.2 and Lemma 1.1, it suffices to show that $\varphi_{\infty} \equiv 1$ if and only if

$$
D\left(\varphi_{\infty}: R^{d}\right)=0 .
$$

The "only if" part is obvious. The proof of the "if" part is given as follows. Suppose $D\left(\varphi_{\infty}: R^{d}\right)=0$ i. e.

$$
\int_{R^{d}} \sum_{i j=1}^{d} a_{i j} \frac{\partial \varphi_{\infty}}{\partial x_{i}} \frac{\partial \varphi_{\infty}}{\partial x_{j}} d x=0 .
$$

Then by the strict positivity of the matrix $A(x)$, one sees that

$$
\frac{\partial \varphi_{\infty}}{\partial x_{i}}=0 \text { a. e., } i=1, \ldots, d \text {. }
$$

This implies that for a mollifier $\phi_{\varepsilon}(x)$ defined by

$$
\phi_{\varepsilon}(x) \equiv \varepsilon^{-d} \phi(x / \varepsilon), \quad \varepsilon>0,
$$

where $\phi \in C_{0}^{\infty}\left(R^{d}\right)$ is non negative, $\int_{R^{d}} \phi(x) d x=1$ and such that $\operatorname{Supp}(\psi) \leqq\left\{x \in R^{d}:|x| \leqq 1\right\}$, one has that the convolution

$$
\varphi_{\infty}^{*} \psi_{\varepsilon}(x)=a \text { constant } M_{\varepsilon}
$$

since $\varphi_{\infty}{ }^{*} \psi_{\mathrm{c}} \in C^{\infty}\left(R^{d}\right)$ and $\frac{\partial\left\{\varphi_{\infty}{ }^{*} \psi_{\mathrm{s}}\right\}}{\partial \mathrm{x}_{i}}=\frac{\partial \varphi_{\infty}}{\partial x_{i}} * \psi_{\mathrm{s}}=0, \quad i=1, \ldots, d$. Furthermore, since $\varphi_{\infty}$ is bounded and continuous,

$$
\lim _{\bullet>0} \varphi_{\infty}^{*} \psi_{c}(x)=\varphi_{\infty}(x), \quad x \in R^{d} .
$$

Combining all of this, it follows that $\varphi_{\infty}$ must be identical with 
the constant 1. This compeltes the proof.

Q. E. D.

We shall now prove our main theorems by means of Theorem 1.1.

Proof of Theorem A. First define the following function

$$
\psi_{\rho}(|x|)=\frac{\int_{|x|}^{\rho} s^{1-d} \bar{E}_{1}^{-1}(s) d s}{\int_{1}^{\rho} s^{1-d} \bar{E}_{1}^{-1}(s) d s} \quad \text { for } 1 \leqq|x| \leqq \rho .
$$

Then it is clear that $\phi_{\rho}(|x|)-\phi_{\rho}(x)$ is absolutely continuous and has bounded partial derivatives on $\Sigma_{\rho}-\bar{\Sigma}_{1}$, since $L$ is uniformly elliptic on the set. Therefore the function $\phi_{\rho}-\phi_{\rho}$ is an element of $H^{1,2}\left(\Sigma_{\rho}\right.$ $\left.-\bar{\Sigma}_{1}\right)$. See Agmon [1], Theorem 3. 1. Furthermore $\phi_{p}-\phi_{\rho}$ vanishes on the boundary $\partial\left(\Sigma_{\rho}-\bar{\Sigma}_{1}\right)$. Hence we see from Theorem 11.5 of Lions and Magenes [15] page 68 that $\phi_{\rho}-\phi_{\rho} \in H_{0}^{1,2}\left(\Sigma_{\rho}-\bar{\Sigma}_{1}\right)$.

Now applying Proposition 1.1 (iii), one obtains

$$
\begin{gathered}
\int_{\Sigma_{\rho}-\Sigma_{1}} \sum_{i, j=1}^{d} a_{i j} \frac{\partial \psi_{\rho}(|x|)}{\partial x_{i}} \frac{\partial \psi_{\rho}(|x|)}{\partial x_{j}} d x \\
\geqq \int_{\Sigma_{\rho}-\Sigma_{1}} \sum_{i, j=1}^{d} a_{i j} \frac{\partial \varphi_{\rho}}{\partial x_{i}} \frac{\partial \varphi_{\rho}}{\partial x_{j}} d x .
\end{gathered}
$$

By a simple computation, the left hand side of the above inequality is equal to

$$
\frac{\left|S^{d-1}\right|}{\int_{1}^{\rho} r^{1-d} \bar{E}_{1}^{-1}(r) d r} \searrow 0, \quad \text { as } \rho \nearrow+\infty
$$

by the assumption. This combined with the above inequality implies that

$$
\lim _{\rho \nearrow+\infty} D\left(\varphi_{\rho}: \Sigma_{\rho}\right)=0
$$

which completes the proof of Theorem A by Theorem 1.1.

Q. E. D.

In order to prove Theorem B, we need the following Lemma.

Lemma 1. 2. For every $v \in C_{0}^{\infty}\left(R^{d}\right)$ such that $v(x)=1$ if $|x| \leqq 1$ 
and $=0$ if $|x| \geqq \rho$, it holds that

$$
D\left(v: \Sigma_{\rho}\right) \geqq \int_{S^{d-1}} d \sigma_{-}^{-} \frac{\left|S^{d-1}\right|}{\int_{1}^{\rho} r^{1-d} E_{2}^{-1}(r \sigma) d r} .
$$

Proof. By the Schwarz inequality,

$$
(x, \xi)^{2} \leqq\left(A^{-1}(x) x, x\right) \cdot(A(x) \xi, \xi)
$$

for every $(x, \xi) \in R^{d} \times R^{d}$.

Using the above inequality, we have

$$
\begin{aligned}
D\left(v: \Sigma_{\rho}\right) & =\int_{\Sigma_{\rho}-\Sigma_{1}} \sum_{i, j=1}^{d} a_{i j} \frac{\partial v}{\partial x_{i}} \frac{\partial v}{\partial x_{j}} d x \\
& \geqq \int_{\Sigma_{\rho}-\Sigma_{1}} \frac{1}{\left(A^{-1}(x) x, x\right)}(x, \nabla v)^{2} d x \\
& =\int_{\Sigma_{\rho}-\Sigma_{1}} E_{2}(x)\left[\frac{\partial v}{\partial n}\right]^{2} d x \\
& =\int_{S^{d-1}}\left|S^{d-1}\right| \cdot d \sigma \int_{1}^{\rho} r^{d-1} E_{2}(r \sigma)\left[\frac{\partial v(r \sigma)}{\partial r}\right]^{2} d r .
\end{aligned}
$$

Applying the Schwarz inequality again, we obtain for every $\sigma \in S^{d-1}$;

$$
\begin{aligned}
& {\left[\int_{1}^{\rho} r^{d-1} E_{2}(r \sigma)\left[\frac{\partial v(r \sigma)}{\partial r}\right]^{2} d r\right] \cdot\left[\int_{1}^{\rho} r^{1-d} E_{2}^{-1}(r \sigma) d r\right]} \\
& \geqq\left[\int_{1}^{\rho} \frac{\partial v(r \sigma)}{\partial r} d r\right]^{2}=[v(\rho \sigma)-v(\sigma)]^{2}=1,
\end{aligned}
$$

from the assumption on $v(x)$. Combining (1.21) and (1.22), we obtain the desired inequality (1.19).

Q. E. D.

Proof of Theorem B. By virtue of Proposition 1.1 (iii), one can choose a sequence $w_{n}, n \geqq 1 \in C_{0}^{\infty}\left(\Sigma_{\rho}-\bar{\Sigma}_{1}\right)$ such that $w_{n}$ converges to $\varphi_{\rho}-\phi_{\rho}$ in $H_{0}^{1,2}\left(\Sigma_{\rho}-\bar{\Sigma}_{1}\right)$. Since $L$ is uniformly elliptic on $\bar{\Sigma}_{\rho}$, the above implies that

$$
\lim _{n>+\infty} D\left(w_{n}+\phi_{\rho}: \Sigma_{\rho}\right)=D\left(\varphi_{\rho}: \Sigma_{\rho}\right)
$$

Applying Lemma. 1.2 to the function $w_{n}+\phi_{\rho}$, we obtain 


$$
D\left(w_{n}+\phi_{\rho}: \Sigma_{\rho}\right) \geqq \int_{S^{d-1}} d \sigma \frac{\left|S^{d-1}\right|}{\int_{1}^{\rho} r^{1-d} E_{2}^{-1}(r \sigma) d r} .
$$

Letting $n \nearrow+\infty$,

$$
\begin{aligned}
D\left(\varphi_{\rho}: \Sigma_{\rho}\right) & \geqq \int_{S^{d-1}} d \sigma_{\frac{1}{\int_{1}^{\rho}} r^{1-d} E_{2}^{-1}(r \sigma) d r} \\
& \geqq \int_{S^{d-1}} d \sigma_{\frac{1}{\int_{1}^{\infty}} r^{1-d} E_{2}^{-1}(r \sigma) d r}>0
\end{aligned}
$$

by the assumption. Thus we have shown

$$
\lim _{\rho>+\infty} D\left(\varphi_{\rho}: \Sigma_{\rho}\right)>0 \text {. }
$$

This together with Theorem 1.1 completes the proof of Theorem B.

Q. E. D.

\section{Chapter 2. Criteria for Recurrence and Transience II.} Hypoelliptic differential operator

In this chapter we shall study the recurrence and transience problems of the diffusion process associated with a degenerate elliptic operator of self adjoint form.

We assume that the self adjoint operator $L=\sum_{i, j=1}^{d} \frac{\partial}{\partial x_{i}}\left(a_{i j} \frac{\partial}{\partial x_{j}}\right)$ under consideration is of the form

$$
L=\frac{1}{2} \sum_{i=1}^{r} X_{i}^{2}+Y, \quad r \leqq d
$$

on $R^{d}$, where

$$
\begin{aligned}
X_{i} & =\sum_{j=1}^{d} g_{j i} \frac{\partial}{\partial x_{j}}, \quad i=1, \ldots, r \\
Y & =\sum_{i=1}^{d} f_{i} \frac{\partial}{\partial x_{i}}
\end{aligned}
$$

and $g_{i j}, f_{i} \in C^{\infty}\left(R^{d}\right), i=1, \ldots, d, j=1, \ldots, r$. Our fundamental assumption on the operator $L$ is

$$
\operatorname{rank} \mathscr{L}\left(X_{1}, \ldots, X_{r}\right)_{x}=d, \quad x \in R^{d},
$$


where $\mathscr{L}\left(X_{1}, \ldots, X_{r}\right)$ is the minimal Lie algebra generated by $X_{i}$, $i=1, \ldots, r$. See Bony [3], pp. 277-279.

Remark 2.1. A simple computation shows that $Y \in \mathscr{L}\left(X_{1}, \ldots, X_{r}\right)$ under the self adjointness of the operator. Therefore the assumption (*) is equivalent to the weaker one

$$
\operatorname{rank} \mathscr{L}\left(X_{1}, \ldots, X_{r}, Y\right)_{x}=d, \quad x \in R^{d} .
$$

Remark 2. 2. Hörmander [9], p. 149, has shown that under the rank condition $(* *)$, the second order differential operator (2.1) which is not necessarily self adjoint is hypoelliptic i. e. if $f$ is a. e. equal to a $C^{\infty}$ function in an open subset $D$ of $R^{d}$, any distribution solution $u$ of $L u=f$ in $D$ is a. e. equal to a $C^{\infty}$ function in $D$.

We now construct a minimal diffusion process in the sense of McKean [17] which is associated with the operator $L$.

From our definition of $L$, we have

$$
a_{i j}=\frac{1}{2} \sum_{k=1}^{r} g_{i k} g_{j k}, \quad i, j=1, \ldots, d
$$

and define

$$
\begin{aligned}
& G(x)=\left(g_{i j}(x)\right)_{\substack{1 \leq i \leq d \\
1 \leq j \leq r}} \\
& b_{i}(x)=\sum_{j=1}^{d} \frac{\partial a_{j i}}{\partial x_{j}}, \quad i=1, \ldots, d \\
& b(x)=\left(b_{i}(x)\right)_{1 \leq i \leq d .}
\end{aligned}
$$

Let $\beta_{t}=\left(\beta_{t}^{i}\right)_{1 \leq i \leq r}$ be an $r$-dimensional Brownian motion.

Consider the stochastic differential equation in $R^{d}$,

$$
X_{t}=X_{0}+\int_{0}^{t} G\left(x_{s}\right) d \beta_{s}+\int_{0}^{t} b\left(x_{s}\right) d s, \quad x_{0} \in R^{d} .
$$

This has a unique continuous solution $x_{t}$ up to the explosion time $\zeta$. Thus we have obtained the diffusion process $\left(X_{t}, \zeta, P_{x}\right), x \in R^{d}$.

Let $P_{t}(x, d y)$ be the transition probability of the above process. Itô's formula implies that

$u\left(t, x_{t}\right)-u\left(0, x_{0}\right)=\sum_{i=1}^{r} \int_{0}^{t} X_{i} u\left(s, x_{s}\right) d \beta_{s}^{i}+\int_{0}^{t}\left(\frac{\partial}{\partial s}+L\right) u\left(s, x_{s}\right) d s$, for $t<\zeta$ 
and $u \in C_{0}^{\infty}\left(R^{+} \times R^{d}\right)$. Since the first term of the right hand side is a martingale with mean 0 , we get

$$
\begin{aligned}
\int_{R^{d}} & P_{t}\left(x_{0}, d y\right) u(t, y)-u\left(0, x_{0}\right) \\
= & \int_{0}^{t} \int_{R^{d}} P_{s}\left(x_{0}, d y\right)\left(\frac{\partial}{\partial s}+L\right) u(s, y) d s .
\end{aligned}
$$

Consequently the diffusion process constructed above is associated with the operator $L$. We note that under our fundamental assumption $(*)$, the transition probability $P_{t}(x, d y)$ has a smooth density i. e. there exists a function $p_{t}(x, y) \in C^{\infty}\left((0, \infty) \times R^{d} \times R^{d}\right)$ such that

$$
\begin{aligned}
& \left(\frac{\partial}{\partial t}-L_{x}\right) p_{t}(x, y)=0 \\
& \left(\frac{\partial}{\partial t}-L_{y}\right) p_{t}(x, y)=0
\end{aligned}
$$

where $L_{x}=\sum_{i, j=1}^{d}-\frac{\partial}{\partial x_{i}}-\left(a_{i j}(x) \frac{\partial}{\partial x_{j}}\right)$ and $L_{y}=\sum_{i, j=1}^{d}-\frac{\partial}{\partial y_{i}}\left(a_{i j}(y) \frac{\partial}{\partial y_{j}}\right) . \quad$ In fact, (2.5) is a special case of Theorem 3 of Ichihara and Kunita [11].

Remark 2.3. Under our assumption (*), the main theorem of Stroock and Varadhan [20], combined with Proposition 2. 1 of Bony [3], states that the topological support of $P_{x}$ coincides with the set of all continuous functions taking values in $R^{d}$ and initial value $x$, i. e.

$$
\operatorname{Supp}\left(P_{x}\right)=C_{x}, \quad x \in R^{d},
$$

where $C_{x}=\left\{\varphi_{t}, t \geqq 0: \varphi_{0}=x, \varphi_{t}\right.$ is continuous on $[0, e(\varphi))$ and where $e(\varphi)$ is the explosion time of $\varphi\}$.

As a direct consequence of this fact, one has the communication property for the process $\left(X_{t}, \zeta, P_{x}\right)$. More precisely, for each $(t, x) \in(0, \infty) \times R^{d}$ and each open subset $U$ of $R^{d}, P_{t}(x, U)>0$.

Remark 2.4. The communication property for the process $\left(X_{t}, \zeta, P_{x}\right)$ implies the following fact which is important for our discussion.

Let $D$ be an open domain with compact closure $\bar{D}$. Define $\tau_{D}^{\prime}=$ $\inf \left\{t \geqq 0 ; x_{t} \notin \bar{D}\right\}$. Then we have 


$$
\sup _{x \in \bar{D}} \mathrm{E}_{x}\left[\tau_{D}^{\prime}\right]<+\infty
$$

See Stroock and Varadhan [21], Remark 5. 2 for the proof.

Denote by $\widetilde{E}_{2}(x)$ the minimal eigenvalue of the matrix $A(x)=$ $\left(a_{i j}(x)\right)_{1 \leqq i, j \leqq d}$. We note that

$$
\widetilde{E}_{2}(x) \leqq E_{2}(x)
$$

if the matrix is not singular at $x$. Thus the condition in Theorem $\mathrm{D}$ below is stronger than the one in Theorem $\mathrm{B}$.

The followings are our main results in this chapter.

Theorem C. If the condition (*) holds and if

$$
\int_{1}^{\infty} r^{1-d} \bar{E}_{1}^{-1}(r) d r=+\infty
$$

then the process is recurrent.

Theorem D. If the condition (*) holds and if

$$
\int_{r_{0}}^{\infty} r^{1-d} \widetilde{E}_{2}^{-1}(r \sigma) d r<+\infty
$$

for some fixed constant $r_{0}>0$ on a subset of $S^{d-1}$ with positive uniform measure, then the process is transient.

The proofs of the above theorems will follow the same lines as in the preceding chapter, though several steps have to be redone.

We start with the Dirichlet problem. The first boundary vaule problem for the most general elliptic differential operator has been treated from the martingale point of view by Stroock and Varadhan [21].

In the following we need a somewhat more precise result and we shall derive it first. In order to do it, we introduce the "barrier" function

$$
\phi_{\rho}(x)=1-E_{x}\left[e^{-\tau_{1} \wedge \sigma_{\rho}}\right]
$$

and define 


$$
\begin{gathered}
\Gamma_{0, \rho}=\left\{x \in \partial\left(\Sigma_{\rho}-\bar{\Sigma}_{1}\right): \lim _{\substack{y \rightarrow x \\
y \in \Sigma_{\rho}-\Sigma_{1}}} \phi_{\rho}(y)=0\right\}, 1<\rho<+\infty \\
\Gamma_{0, \infty}=S^{d-1} \cap \bigcup_{\rho>1} \Gamma_{0, \rho}
\end{gathered}
$$

Proposition 2. 1. For each $\rho \in(1, \infty]$, (i) $\varphi_{\rho} \in C^{\infty}\left(\Sigma_{\rho}-\bar{\Sigma}_{1}\right)$ and $L \varphi_{\rho}=0$ in $\Sigma_{\rho}-\bar{\Sigma}_{1}$

(ii) $\varphi_{\rho}(y) \stackrel{\dot{\psi}_{\rho}}{\rightarrow} g_{\rho}(x) \equiv \begin{cases}1 & \text { if }|x|=1 \\ 0 & \text { if }|x|=\rho<+\infty\end{cases}$ as $y \in \Sigma_{\rho}-\bar{\Sigma}_{1} \rightarrow x \in \Gamma_{0, p}$

where $\varphi_{\rho}(y) \stackrel{\varphi_{\rho}}{\rightarrow} g_{\rho}(x)$ means that for $\rho<+\infty, \varphi_{\rho}\left(y_{n}\right) \rightarrow g_{\rho}(x)$ if $y_{n, n \geqq 1}$ $\in \Sigma_{\rho}-\bar{\Sigma}_{1}$ tends to $x \in \Gamma_{0, \rho}$ and $\lim \psi_{\rho}\left(y_{n}\right)=0$ and that for $\rho=+\infty$, $\varphi_{\infty}\left(y_{n}\right) \rightarrow g_{\infty}(x) \equiv 1$ if $y_{n, n \geqq 1} \in R^{d}-\bar{\Sigma}_{1}^{n \lambda+\infty}$ tends to $x \in \Gamma_{0, \infty}$ and $\lim _{n>+\infty} \phi_{\rho}\left(y_{n}\right)=0$ for every $\rho \in\left(1, \rho_{0}\right)$ with a positive constant $\rho_{0}=\rho_{0}(x)>1$.

Proof. We first discuss the case $1<\rho<+\infty$. Let $D$ be a domain of $R^{d}$. Stroock and Varadhan [21], Chapter 5 and Theorem 7.1 have formulated the first boundary value problem

$$
\text { "Lu=f in } D
$$

and

$$
u=g \text { on } \partial D^{\prime \prime}
$$

in two ways, which are called the $\tau^{\prime}$ and $\tau$-first boundary value problem respectively, and shown that under our basic assumption (*), there exists a unique solution of each problem if $D$ is bounded and that both the solutions are the same a.e. in $D$. They have further proved that the $\tau^{\prime}$-solution is a weak solution of $L u=f$ in $D$. [21] Corollary 8.2. Since $\varphi_{\rho}$ is a unique solution of the $\tau^{\prime}$-first boundary value problem with respect to $D=\Sigma_{\rho}-\bar{\Sigma}_{1}, f \equiv 0$ and $g=\left\{\begin{array}{ll}1 & \text { on }|x|=1 \\ 0 & \text { on }|x|=\rho\end{array}\right.$, see [21]. Theorem 5.2, this combined with the above facts implies that $\varphi_{\rho}$ is also a weak solution of $L u=0$ in $\Sigma_{\rho}-\bar{\Sigma}_{1}$, i. e.

$$
\int_{\Sigma_{\rho}-\Sigma_{1}} \varphi_{\rho} \cdot L \psi d x=0 \text { for all } \phi \in C_{0}^{\infty}\left(\Sigma_{\rho}-\bar{\Sigma}_{1}\right) \text {. }
$$

By virtue of the hypoellipticity of the operator $L$ Remark 2.2, there exists a function $\psi_{\rho} \in C^{\infty}\left(\Sigma_{\rho}-\bar{\Sigma}_{1}\right)$ such that $\psi_{\rho}$ is a. e. equal to $\varphi_{\rho}$ in 
$\Sigma_{\rho}-\bar{\Sigma}_{1}$ and $L \phi_{\rho}=0$.

We want to show that $\varphi_{\rho}(x)=\phi_{\rho}(x)$ for all $x \in \Sigma_{\rho}-\bar{\Sigma}_{1}$. In order to do this, we note that the transition probability of the stopped process $x_{t}, t<\tau_{1} \wedge \sigma_{\rho}$ is absolutely continuous with respect to the Lebesgue measure $d y$ because the transition probability $P_{t}(x, d y)$ of the process $\left(X_{t}, \zeta, P_{x}\right)$ has the smooth density $p_{t}(x, y)$ with respect to the Lebesgue measure $d y$ and

$$
\begin{aligned}
P_{t}^{o}(x, d y) & =P_{x}\left\{x_{t} \in d y, t<\tau_{1} \wedge \sigma_{\rho}\right\} \\
& \leqq P_{x}\left\{x_{t} \in d y, t<\zeta\right\}=P_{t}(x, d y) .
\end{aligned}
$$

Returning to the proof of Proposition 2.1, we first note that $\varphi_{\rho}\left(x_{t \wedge \tau_{1} \wedge \sigma_{\rho}}\right)$ is a $P_{x}$-martingale, i. e.

$$
E_{x}\left[\varphi_{\rho}\left(x_{t \wedge \tau_{1} \wedge \sigma_{\rho}}\right)\right]=\varphi_{\rho}(x), x \in \Sigma_{\rho}-\bar{\Sigma}_{1},
$$

holds. Decompose the left hand side as

$$
\begin{aligned}
& E_{x}\left[\varphi_{\rho}\left(x_{t \wedge \tau_{1} \wedge \sigma_{\rho}}\right)\right]=E_{x}\left[\varphi_{\rho}\left(x_{t}\right): t<\tau_{1} \wedge \sigma_{\rho}\right] \\
& \quad+E_{x}\left[\varphi_{\rho}\left(x_{\tau_{1} \wedge \sigma_{\rho}}\right): t \geqq \tau_{1} \wedge \sigma_{\rho}\right] .
\end{aligned}
$$

The first term equals $E_{x}\left[\psi_{\rho}\left(x_{t}\right): t<\tau_{1} \wedge \sigma_{\rho}\right]$ by the absolute continuity of $P_{t}^{\rho}(x, d y)$. Since $\psi_{\rho}\left(x_{t}\right)$ converges to $\psi_{\rho}(x)$ as $t \searrow 0$ and $\lim _{t \searrow 0} P_{x}\left[t<\tau_{1} \wedge \sigma_{\rho}\right]=1$, one obtains that

$$
\lim _{t>0} E_{x}\left[\psi_{\rho}\left(x_{t}\right): t<\tau_{1} \wedge \sigma_{\rho}\right]=\psi_{\rho}(x) .
$$

The second term in the right hand side of (2.10) satisfies

$$
\begin{aligned}
& E_{x}\left[\varphi_{\rho}\left(x_{\tau_{1} \wedge \sigma_{\rho}}\right): t \geqq \tau_{1} \wedge \sigma_{\rho}\right] \\
& \leqq P_{x}\left[t \geqq \tau_{1} \wedge \sigma_{\rho}\right] \searrow 0
\end{aligned}
$$

as $t \searrow 0$. Consequently it follows that

$$
\begin{aligned}
& \lim _{t \backslash 0} E_{x}\left[\varphi_{\rho}\left(x_{t \wedge \tau_{1} \wedge \sigma_{\rho}}\right)\right] \\
= & \lim _{t \backslash 0} E_{x}\left[\varphi_{\rho}\left(x_{t}\right): t<\tau_{1} \wedge \sigma_{\rho}\right] \\
= & \lim _{t \backslash 0} E_{x}\left[\phi_{\rho}\left(x_{t}\right): t<\tau_{1} \wedge \sigma_{\rho}\right]=\psi_{\rho}(x),
\end{aligned}
$$

which shows that $\varphi_{\rho}$ is identical with $\psi_{\rho}$ in $\Sigma_{\rho}-\bar{\Sigma}_{1}$. The boundary condition (ii) has been already shown by Stroock and Varadhan [21], Theorem 5. 2.

For $\rho=+\infty$, [etting $\rho \nearrow+\infty$, one sees that 


$$
\int_{R^{d}-\Sigma_{1}} \varphi_{\infty} \cdot L \phi d x=0 \text { for all } \psi \in C_{0}^{\infty}\left(R^{d}-\bar{\Sigma}_{1}\right)
$$

from (2.9) and the fact that $\varphi_{\rho}$ is boundedly convergent to $\varphi_{\infty}$ on $R^{d}$. This implies that $\varphi_{\infty}$ is a weak solution of $L u=0$ in $R^{d}-\bar{\Sigma}_{1}$. In order to obtain the smoothness of $\varphi_{\infty}$, we need the following Harnack inequality, valid under the condition $(*)$.

Harnack inequality (Bony [2], Theorem 7.2). If $u$ is a positive solution of $L u=0$ in an open domain $D$, then for any compact subset $K$ of $D$, any point $y_{0} \in D$ and any multi index $p=\left(p_{1}, \ldots, p_{d}\right)$ of derivatives, there exists a positive constant $C=C\left(K, y_{0}, p\right)$ independent of $u$ such that

$$
\sup _{x \in K}\left|\frac{\partial^{|p|} u(x)}{\partial x_{1}^{p_{1}} \ldots \partial x_{d}^{p^{d}}}\right| \leqq C \cdot u\left(y_{0}\right)
$$

where $|p|=\sum_{i=1}^{d} p_{i}$

It is easily derived from Bony's Harnack inequality that for any compact subset $K$ of $\Sigma_{\rho_{0}}-\bar{\Sigma}_{1}$ with $\rho_{0}>1$, there exists a positive constant $C=C\left(K, \rho_{0}\right)$ such that

$$
\left|\varphi_{\rho}(x)-\varphi_{\rho}\left(x^{\prime}\right)\right| \leqq C\left|x-x^{\prime}\right|
$$

for every $x, x^{\prime} \in K$ and $\rho \in\left[\rho_{0},+\infty\right)$. Since $\varphi_{\infty}(x)=\lim _{\rho>+\infty} \varphi_{\rho}(x)$, $x \in R^{d}$, we obtain

$$
\left|\varphi_{\infty}(x)-\varphi_{\infty}\left(x^{\prime}\right)\right| \leqq C\left|x-x^{\prime}\right|, \text { for } x, x^{\prime} \in K .
$$

This implies that $\varphi_{\infty}$ is continuous in $R^{d}-\bar{\Sigma}_{1}$. Again using the hypoellipticity of $L$, we see that $\varphi_{\infty}$ is a genuine solution of $L u=0$.

The boundary condition for $\varphi_{\infty}$ follows from the fact that $\varphi_{\rho} \nearrow \varphi_{\infty}$ $\begin{array}{ll}\text { as } \rho \nearrow+\infty . & \text { Q. E. D. }\end{array}$

Corollary 2.1. For any ball $U \subset R^{d}, \varphi_{U}(x) \equiv P_{x}\left[x_{t} \in \bar{U}\right.$ for some $t>0] \in C^{\infty}\left(R^{d}-\bar{U}\right), L \varphi_{U}=0$ in $R^{d}-\bar{\Sigma}_{1}$ and furthermore $\varphi_{U}$ is strictly positive on $R^{d}$. 
Proof. The assertions except for the last one follow as after Corollary 1.1. The strict positivity of $\varphi_{U}$ follows from Remark 2.3. Q.E. D.

The martingale method used in Lemma 1.1 combined with Corollary 2.1 yields the following

Corollary 2. 2. The process $\left(X_{t}, p_{x}\right)$ is recurrent if and only if $\varphi_{\infty} \equiv 1$ on $R^{d}$.

Using a technique similar to that of Corollary 1.2, we can prove

Corollary 2. 3. If the process is transient, then $\varphi_{\infty}$ cannot attain the maximum value 1 in the exterior domain $R^{d}-\bar{\Sigma}_{1}$ and

$$
P_{x}\left[\lim _{t / 6}\left|x_{t}\right|=+\infty\right] \equiv 1 \text { on } R^{d} \text {. }
$$

Finally we summarize the results of [21] on the set of regular points. Let $\Gamma_{\rho}$ be the set of all regular points with respect to $\left(X_{t}, \zeta, P_{x}\right)$ and $\Sigma_{p}-\bar{\Sigma}_{1}$ i. e.

$$
\Gamma_{\rho}=\left\{x \in \partial\left(\Sigma_{\rho}-\bar{\Sigma}_{1}\right): P_{x}\left[\tau_{\Sigma_{\rho}-\Sigma_{1}}=0\right]=1\right\}
$$

where $\tau_{\Sigma_{\rho-\Sigma_{1}}}=\inf \left\{t \geqq 0 ; x_{t} \in\left(\overline{{\overline{\Sigma_{\rho}}}_{-\bar{\Sigma}_{1}}}\right)^{c}\right\}$, and let

$$
\Gamma_{1, \rho}=\left\{x \in \partial\left(\Sigma_{\rho}-\bar{\Sigma}_{1}\right): \sum_{i, j=1}^{d} a_{i j}(x) x_{t} x_{j}>0\right\} .
$$

Stroock and Varadhan [21], Remark 5.4 and Theorem 6.3 have shown for our case that

$$
\Gamma_{1, \rho} \leqq \Gamma_{\rho} \leqq \Gamma_{0, \rho} \text { for } 1<\rho<+\infty .
$$

The following lemma shows that the set $\Gamma_{\rho}$ is large enough.

Lemma 2. 2. $\Gamma_{1, p}$ is open dense in $\partial\left(\Sigma_{\rho}-\bar{\Sigma}_{1}\right)$.

For the proof of this lemma, we introduce the following notation. For a given $Z \in \mathscr{L}\left(X_{1}, \ldots, X_{r}\right)$ where $Z=\sum_{i=1}^{d} z_{i}(x)-\frac{\partial}{\partial x_{i}}, z_{i} \in C^{\infty}\left(R^{d}\right)$, 
we denote by $Z(x)$ the vector $\left(z_{i}(x)\right)_{1 \leqq i \leqq d}$.

Proof of Lemma 2.2. Suppose there exists an open subset $U$ of $\{|x|=\bar{\rho}\}$ where $\bar{\rho}=1$ or $\rho(<+\infty)$ such that

$$
\sum_{i, j=1}^{d} a_{\imath}(x) x_{\imath} x_{\jmath}=0 \text { for all } x \in U .
$$

Then it is easy to see that the inner product

$$
\left(x, X_{i}(x)\right)=0 \text { on } U, i=1, \ldots, r \text {. }
$$

By virtue of Theorem 2.2 of Bony [3], one has that

$$
(x, Z(x))=0 \text { on } U \text { for all } Z \in \mathscr{L}\left(x_{1}, \ldots, x_{r}\right)
$$

which is a contradiction since the Lie algebra $\mathscr{L}\left(X_{1}, \ldots, X_{r}\right)$ has the full rank " $d$ ". Therefore $\Gamma_{1, \rho}$ must be open dense in $\partial\left(\Sigma_{\rho}-\bar{\Sigma}_{1}\right)$.

Q. E. D.

Our next task is to extend the variational principle to the degenerate case. In order to do this we make use of the Hilbert space $H_{0}^{1,2}\left(\Sigma_{\rho}-\bar{\Sigma}_{1}: d x\right)$.

From the definition of the $(d \times r)$-matrix $G(x)$ it is clear that $A(x)=\frac{1}{2}\left(G \cdot G^{t}\right)(x)$ where $G^{t}$ is the transpose of $G$. Using a standard technique in functional analysis (Yosida [22], page 58), we can obtain the following

Lemma 2. 3. Each $u \in H_{0}^{1,2}\left(\Sigma_{\rho}-\bar{\Sigma}_{1}: d x\right)$ has the following properties: (i) $u$ and $\left(G^{t} \nabla u\right)_{i} \in L^{2}\left(\Sigma_{\rho}-\bar{\Sigma}_{1}\right), i=1, \ldots, r$ where $\nabla u=\left(\frac{\partial u}{\partial x_{i}}\right)_{1 \leqq i \leqq d}$, $\frac{\partial u}{\partial x_{i}}$ represents the distribution derivative.

(ii)

$$
\|u\|_{H_{0}^{1,2}\left(\Sigma_{\rho}-\Sigma_{1}: d x\right)}=\sqrt{\int_{\Sigma_{\rho}-\Sigma_{1}}\left\{u(x)^{2}+\frac{1}{2}\left(G^{t} \nabla u, G^{t} \nabla u\right)(x)\right\} d x} .
$$

We are now ready to state the main proposition.

Proposition 2. 2. For each $\rho \in(1,+\infty)$, let $\phi_{\rho}$ be a $C^{\infty}$ function 
as in Proposition 1.1 (iii), then

(i ) $\varphi_{\rho} \in H_{0}^{1,2}\left(\Sigma_{\rho}-\bar{\Sigma}_{1}: d x\right)+\left\{\phi_{\rho}\right\}$

and

(ii) $\varphi_{\rho}$ minimizes an integral

$$
\int_{\Sigma_{\rho}-\Sigma_{1}}\left(G^{t} \nabla u, G^{t} \nabla u\right) d x
$$

over all $u \in H_{0}^{1,2}\left(\Sigma_{\rho}-\bar{\Sigma}_{1}: d x\right)+\left\{\phi_{\rho}\right\}$.

Note that $H_{0}^{1,2}\left(\Sigma_{\rho}-\bar{\Sigma}_{1}: d x\right)+\left\{\phi_{\rho}\right\}$ is independent of the choice of $\phi_{\rho}$ and increasing as in the remark after Proposition 1.1.

Our proof is based on a perturbation method. Let $X_{t}^{i}, \varepsilon>0$ be the minimal diffusion process corresponding to $L^{i} \equiv L+\varepsilon \Delta_{d}$ where $\Delta_{d}=$ $\sum_{i=1}^{d} \frac{\partial^{2}}{\partial x_{i}^{2}}$ and $\tau_{1}^{e}, \sigma_{\rho}^{e}$ and $\varphi_{\rho}^{e}$ be defined as before. Under our basic assumption on the operator $L$, we have the following lemma which plays an essential role in the proof of Proposition 2.2.

Lemma 2.4 (Stroock and Varadhan [21], Theorems 7. 1, 9.2, and Remark 9.2). For each $\rho \in(1,+\infty)$, one has that

$$
\lim _{i>0} \varphi_{\rho}^{i}(x)=\varphi_{\rho}(x) \text { a.e. in } \Sigma_{\rho}-\bar{\Sigma}_{1} \text {. }
$$

Proof of Proposition 2.2. By virtue of the variational principle for the nondegenerate case, one has that

$$
\int_{\Sigma_{\rho}-\Sigma_{1}}\left\{\varepsilon\left(\nabla \varphi_{\rho}^{e}, \nabla \varphi_{\rho}^{s}\right)+\frac{1}{2}\left(G^{t} \nabla \varphi_{\rho}^{e}, G^{t} \nabla \varphi_{\rho}^{e}\right)\right\} d x
$$

is nonincreasing as $\varepsilon \searrow 0$. Hence its limit exists. Set

$$
\tilde{\varphi}_{\rho}^{\mathrm{s}}=\varphi_{\rho}^{\mathrm{s}}-\phi_{\rho} \text { and } \tilde{\varphi}_{\rho}=\varphi_{\rho}-\phi_{\rho} .
$$

Then it follows from Proposition 1.1 (iii) that

$$
\tilde{\varphi}_{\rho}^{e} \in H_{0}^{1,2}\left(\Sigma_{\rho}-\bar{\Sigma}_{1}\right) \leqq H_{0}^{1,2}\left(\Sigma_{\rho}-\bar{\Sigma}_{1}: d x\right) .
$$

We have, for $\varepsilon>\varepsilon^{\prime}>0$

$$
\int_{\Sigma_{\rho}-\Sigma_{1}} \frac{1}{2}\left(G^{t} V\left(\tilde{\varphi}_{\rho}^{\varepsilon}-\tilde{\varphi}_{\rho}^{\varepsilon^{\prime}}\right), G^{t} V\left(\tilde{\varphi}_{\rho}^{\varepsilon}-\widetilde{\varphi}_{\rho}^{\varepsilon^{\prime}}\right)\right) d x
$$




$$
\begin{aligned}
& \int_{\Sigma_{\rho}-\varepsilon_{1}} \frac{1}{2}\left(G^{t} \nabla\left(\varphi_{\rho}^{\varepsilon}-\varphi_{\rho}^{\varepsilon^{\prime}}\right), G^{t} \nabla\left(\varphi_{\rho}^{\varepsilon}-\varphi_{\rho}^{\varepsilon^{\prime}}\right)\right) d x \\
& \leqq \int_{\Sigma_{\rho}-\varepsilon_{1}}\left\{\varepsilon^{\prime}\left(\nabla\left(\varphi_{\rho}^{\varepsilon}-\varphi_{\rho}^{\varepsilon^{\prime}}\right), \nabla\left(\varphi_{\rho}^{\varepsilon}-\varphi_{\rho}^{\varepsilon^{\prime}}\right)\right)\right. \\
&\left.\quad+\frac{1}{2}\left(G^{t} \nabla\left(\varphi_{\rho}^{\varepsilon}-\varphi_{\rho}^{\varepsilon^{\prime}}\right), G^{t} \nabla\left(\varphi_{\rho}^{\varepsilon}-\varphi_{\rho}^{\varepsilon^{\prime}}\right)\right)\right\} d x .
\end{aligned}
$$

By the same reasoning as in Proposition 1.2, this equals

$$
\begin{aligned}
& \int_{\Sigma_{\rho}-\Sigma_{1}}\left\{\varepsilon^{\prime}\left(\nabla \varphi_{\rho}^{\varepsilon}, \nabla \varphi_{\rho}^{\varepsilon}\right)+\frac{1}{2}\left(G^{t} \nabla \varphi_{\rho}^{\mathrm{c}}, G^{t} \nabla \varphi_{\rho}^{\mathrm{e}}\right)\right\} d x \\
& -\int_{\Sigma_{\rho}-\Sigma_{1}}\left\{\varepsilon^{\prime}\left(\nabla \varphi_{\rho}^{\varepsilon^{\prime}}, \nabla \varphi_{\rho}^{\varepsilon^{\prime}}\right)+\frac{1}{2}\left(G^{t} \nabla \varphi_{\rho}^{\varepsilon^{\prime}}, G^{t} \nabla \varphi_{\rho}^{\varepsilon^{\prime}}\right)\right\} d x \\
& \leqq \\
& \int_{\Sigma_{\rho}-\Sigma_{1}}\left\{\varepsilon\left(\nabla \varphi_{\rho}^{\varepsilon}, \nabla \varphi_{\rho}^{\varepsilon}\right)+\frac{1}{2}\left(G^{t} \nabla \varphi_{\rho}^{\varepsilon}, G^{t} \nabla \varphi_{\rho}^{\varepsilon}\right)\right\} d x \\
& -\int_{\Sigma_{\rho}-\Sigma_{1}}\left\{\varepsilon^{\prime}\left(\nabla \varphi_{\rho}^{\varepsilon^{\prime}}, \nabla \varphi_{\rho}^{\varepsilon^{\prime}}\right)+\frac{1}{2}\left(G^{t} \nabla \varphi_{\rho}^{\varepsilon^{\prime}}, G^{t} \nabla \varphi_{\rho}^{c^{\prime}}\right)\right\} d x
\end{aligned}
$$

which tends to zero as $\varepsilon, \varepsilon^{\prime} \searrow 0$.

Combining this fact with Lemma 2.4, one obtains

$$
\lim _{\varepsilon, \varepsilon^{\prime} \backslash 0}\left\|\tilde{\varphi}_{\rho}^{\varepsilon}-\tilde{\varphi}_{\rho}^{\varepsilon^{\prime}}\right\|_{H_{0}^{1,2}\left(\Sigma_{\rho}-\Sigma_{1}: d x\right)}=0 .
$$

Therefore there exists a unique element $\tilde{\varphi}$ of $H_{0}^{1,2}\left(\Sigma_{\rho}-\bar{\Sigma}_{1}: d x\right)$ to which $\tilde{\varphi}_{\rho}^{\varepsilon}$ converges as $\varepsilon \searrow 0$. On the other hand, $\tilde{\varphi}_{\rho}^{\varepsilon}$ itself converges to $\tilde{\varphi}_{\rho}$ a. e. in $\Sigma_{\rho}-\bar{\Sigma}_{1}$ by Lemma 2.4. This implies that $\tilde{\varphi}_{\rho}$ has to be in $H_{0}^{1,2}\left(\Sigma_{\rho}-\bar{\Sigma}_{1} ; d x\right)$ and that $\tilde{\varphi}_{\rho}=\tilde{\varphi}$ in this space. This completes the proof of the first assertion.

Since $\varphi_{\rho}$ is a weak solution of $L u=0$ in $\Sigma_{\rho}-\bar{\Sigma}_{1}$,

$$
\int_{\Sigma_{\rho}-\Sigma_{1}}\left(G^{t} \nabla \varphi_{\rho}, G^{t} \nabla u\right) d x=0
$$

for every $u \in C_{0}^{\infty}\left(\Sigma_{\rho}-\bar{\Sigma}_{1}\right)$. Taking the closure in the space $H_{0}^{1,2}\left(\Sigma_{\rho}-\bar{\Sigma}_{1}\right.$; $d x)$, one obtains that

$$
\int_{\Sigma_{\rho}-\Sigma_{1}}\left(G^{t} \nabla \varphi_{\rho}, G^{t} \nabla u\right) d x=0
$$

for every $u \in H_{0}^{1,2}\left(\Sigma_{\rho}-\bar{\Sigma}_{1}: d x\right)$. Coming to the proof of the minimality, 
for $\varphi \in H_{0}^{1,2}\left(\Sigma_{\rho}-\bar{\Sigma}_{1} ; d x\right)+\left\{\phi_{\rho}\right\}$

$$
\begin{aligned}
& \int_{\Sigma_{\rho}-\Sigma_{1}}\left(G^{t} \nabla \varphi, G^{t} \nabla \varphi\right) d x \\
= & \int_{\Sigma_{\rho}-\Sigma_{1}}\left(G^{t} \nabla\left\{\varphi_{\rho}+\varphi-\varphi_{\rho}\right\}, G_{t} \nabla\left\{\varphi_{\rho}+\varphi-\varphi_{\rho}\right\}\right) d x \\
= & \int_{\Sigma_{\rho}-\Sigma_{1}}\left(G^{t} \nabla \varphi_{\rho}, G_{t} \nabla \varphi_{\rho}\right) d x+2 \int_{\Sigma_{\rho}-\Sigma_{1}}\left(G^{t} \nabla \varphi_{\rho}, G^{t} \nabla\left(\varphi-\varphi_{\rho}\right)\right) d x \\
+ & \int_{\Sigma_{\rho}-\Sigma_{1}}\left(G^{t} \nabla\left(\varphi-\varphi_{\rho}\right), G^{t} \nabla\left(\varphi-\varphi_{\rho}\right)\right) d x .
\end{aligned}
$$

Since $\varphi-\varphi_{\rho} \in H_{0}^{1,2}\left(\Sigma_{\rho}-\bar{\Sigma}_{1} ; d x\right)$, the second term vanishes (See (2.20)). Hence the following inequality holds.

$$
\int_{\Sigma_{\rho}-\Sigma_{1}}\left(G^{t} \nabla \varphi, G^{t} \nabla \varphi\right) d x \geqq \int_{\Sigma_{\rho}-\Sigma_{1}}\left(G^{t} \nabla \varphi_{\rho}, G^{t} \nabla \varphi_{\rho}\right) d x
$$

which shows that the second assertion is valid. Q. E. D.

Following the same argument as in the preceding chapter, we can obtain the same results for the degenerate case as in Chapter 1 . These are summarized in the foollwing.

Theorem 2.1. ( i )

(2. 22) $\lim _{\rho>+\infty} D\left(\varphi_{\rho}: \Sigma_{\rho}\right)=D\left(\varphi_{\infty}: R^{d}\right)<+\infty$.

(ii) The process $\left(X_{t}, P_{2}\right)$ is recurrent if and only if

$$
\lim _{\rho>+\infty} D\left(\varphi_{\rho}: \Sigma_{\rho}\right)=0 \text {. }
$$

Proof. For the completion of the proof, it suffices to show that $\varphi_{\infty} \equiv 1$ on $R^{d}$ if and only if $D\left(\varphi_{\infty}: R^{d}\right)=0$. The "only if" part is obvious. Suppose $D\left(\varphi_{\infty}: R^{d}\right)=0$

$$
\begin{aligned}
D\left(\varphi_{\infty}: R^{d}\right) & =\int_{R^{d}-\Sigma_{1}} \frac{1}{2}\left(G^{t} \nabla \varphi_{\infty}, G^{t} \nabla \varphi_{\infty}\right) d x \\
& =\int_{R^{d}-\Sigma_{1}} \frac{1}{2} \sum_{i=1}^{r}\left[X_{i} \varphi_{\infty}\right](x) d x .
\end{aligned}
$$


Since $X_{i} \varphi_{\infty}$ is continuous by Proposition 2. 1 ,

$$
X_{i} \varphi_{\infty}(x)=0 \text { in } R^{d}-\bar{\Sigma}_{1}, \quad i=1, \ldots, r .
$$

This leads to

$$
Z \varphi_{\infty}(x) \equiv 0 \text { in } R^{d}-\bar{\Sigma}_{1}
$$

for every $Z \in \mathscr{L}\left(X_{1}, \ldots, X_{r}\right)$. Since $\operatorname{rank} \mathscr{L}\left(X_{1}, \ldots, X_{r}\right)=d$, one obtains

$$
\varphi_{\infty} \equiv a \text { constant in } R^{d}-\bar{\Sigma}_{1}
$$

Taking the boundary value into consideration (see Proposition 2.1 (ii), (2.14) and Lemma 2.2), we conclude that the above constant is equal to 1 . This completes the proof. Q.E.D.

Using Theorem 2.1, we are now able to prove Theorems $\mathrm{C}$ and D. The proof of Theorem $\mathrm{C}$ is carried out in exactly the same way as in Chapter 1 . Note that the space $H_{0}^{1,2}\left(\Sigma_{\rho}-\bar{\Sigma}_{1} ; d x\right)$ in place of $H_{0}^{1,2}\left(\Sigma_{p}-\bar{\Sigma}_{1}\right)$ wll be used in the proof and that $\bar{E}_{1}(r)$ is bounded away from zero on bounded intervals by virtue of Lemma 2. 2 .

In order to prove Theorem $\mathrm{D}$, we need the following lemma similar to Lemma 1.2. Let $\Omega$ be the subset of $S^{d-1}$ mentioned in Theorem D. Without loss of generality, we shall take $r_{0}=1$.

Lemma 2.4. Let a function $v$ be as in Lemma 1.2, then

$$
D\left(v: \Sigma_{\rho}\right) \geqq \int_{\Omega} d \sigma \frac{\left|S^{d-1}\right|}{\int_{1}^{\rho} r^{1-d} \widetilde{E}_{2}^{-1}(r \sigma) d r}
$$

for each $\rho \in(1,+\infty)$.

Proof. From the definition, we have

$$
D\left(v: \Sigma_{\rho}\right) \geqq \int_{\Sigma_{\rho}-\Sigma_{1}} \widetilde{E}_{2}(x) \sum_{i=1}^{d}\left[\frac{\partial v}{\partial x_{i}}\right]^{2} d x .
$$

Using polar coordinates,

$$
D\left(v: \Sigma_{\rho}\right) \geqq \int_{S^{d-1}}\left|S^{d-1}\right| d \sigma \int_{1}^{\rho} r^{d-1} \widetilde{E}_{2}(r \sigma)\left[\frac{\partial v(r \sigma)}{\partial r}\right]^{2} d r
$$




$$
\geqq\left|S^{d-1}\right| \int_{\Omega} d \sigma \int_{1}^{\rho} r^{d-1} \widetilde{E}_{2}(r \sigma)\left[\frac{\partial v(r \sigma)}{\partial r}\right]^{2} d r .
$$

Applying the Schwarz inequality for each $\sigma \in \Omega$, we have

$$
\int_{1}^{\rho} r^{d-1} \widetilde{E}_{2}(r \sigma)\left[\frac{\partial v(r \sigma)}{\partial r}\right]^{2} d r \geqq \frac{(v(r \sigma)-v(\sigma))^{2}}{\int_{1}^{\rho} r^{1-d} \widetilde{E}_{2}^{-1}(r \sigma) d r} .
$$

(2. 25) now follows from (2.26) and (2.27).

Q. E. D.

Proof of Theorem D. Since $\varphi_{\rho}-\phi_{\rho} \in H_{0}^{1,2}\left(\Sigma_{\rho}-\bar{\Sigma}_{1} ; d x\right)$, see Proposition 2. 2(i), there exists a sequence $w_{n}, n \geqq 1$ such that $w_{n} \in C_{0}^{\infty}\left(\Sigma_{\rho}\right.$ $\left.-\bar{\Sigma}_{1}\right)$ and

$$
\lim _{n \lambda+\infty}\left\|w_{n}-\left(\varphi_{\rho}-\phi_{\rho}\right)\right\|_{H_{0}^{1,2}\left(\Sigma_{\rho}-\Sigma_{1}: d x\right)}=0
$$

This gives that

$$
\lim _{\rho>+\infty} D\left(w_{n}+\phi_{\rho}: \Sigma_{\rho}\right)=D\left(\varphi_{\rho}: \Sigma_{\rho}\right)
$$

Applying Lemma 2.4 to $w_{n}+\phi_{\rho}$, we obtain

$$
D\left(w_{n}+\phi_{\rho}: \Sigma_{\rho}\right) \geqq \int_{\Omega} d \sigma \frac{\left|S^{d-1}\right|}{\int_{1}^{\rho} r^{1-d} \tilde{E}_{2}^{-1}(r \sigma) d r} .
$$

As $n \nearrow+\infty,(2.28)$ gives

$$
\begin{aligned}
D\left(\varphi_{\rho}: \Sigma_{\rho}\right) & \geqq \int_{\Omega} d \sigma \frac{\left|S^{d-1}\right|}{\int_{1}^{\rho} r^{1-d} \widetilde{E}_{2}^{-1}(r \sigma) d r} \\
& \geqq \int_{\Omega} d \sigma \frac{\left|S^{d-1}\right|}{\int_{1}^{\infty} r^{1-d} \widetilde{E}_{2}^{-1}(r \sigma) d r}>0,
\end{aligned}
$$

from the assumption in Theorem D. Thus we have shown that

$$
\lim _{\rho>+\infty} D\left(\varphi_{\rho}: \Sigma_{\rho}\right)>0
$$

This together with Theorem 2.1 completes the proof of Theorem D. Q. E. D. 


\section{Chapter 3. Some Examples and Comparison with Friedman [7] and Hasminskii [8]}

We first give some examples to illustrate our main results.

Examples 1 (Recurrent case). If $E_{1}(x)=O\left(\frac{\log |x|}{|x|^{d-2}}\right)$ as $|x| \nearrow+\infty$, then the associated diffusion is recurrent. In particular, if

$$
\max _{i, j=1, \ldots, d}\left|a_{i,}(x)\right|=O\left(\frac{\log |x|}{|x|^{d-2}}\right)
$$

holds, then the above condition is fulfilled.

The followings are the examples of recurrent diffusion processes corresponding to hypoelliptic operators.

(i) $(d=2)$. Let a diffusion $\left(X_{t}, P_{x}\right)$ be associated with

$$
L=\frac{1}{2}\left\{\frac{\partial^{2}}{\partial x_{1}^{2}}+\frac{x_{1}^{2}}{1+x_{1}^{2}} \frac{\partial^{2}}{\partial x_{2}^{2}}\right\} .
$$

Define first order partial differential operators $X_{1}$ and $X_{2}$ by

$$
X_{1}=\frac{\partial}{\partial x_{1}}, \quad X_{2}=\frac{x_{1}}{\sqrt{1+x_{1}^{2}}} \frac{\partial}{\partial x_{2}} .
$$

Then it is easy to see that rank $\mathscr{L}\left(X_{1}, X_{2}\right)_{x}=2, x \in R^{2}$. The operator $L$ satisfies the inequality (3.1), so the diffusion is recurrent.

(ii) $(d=3)$. Let a differential operator on $R^{3}$ be defined by

$$
L=\sum_{i, j=1}^{3} \frac{\partial}{\partial x_{1}}\left(a_{i j} \frac{\partial}{\partial x_{j}}\right)
$$

where

$$
\left\{\begin{array}{l}
a_{11}(x)=\frac{1}{2} \frac{\log \left(2+|x|^{2}\right)}{\left(2+|x|^{2}\right)^{1 / 2}} \\
a_{12}(x)=a_{21}(x)=0 \\
a_{22}(x)=\frac{1}{2} \frac{x_{1}^{2} \log \left(2+|x|^{2}\right)}{\left(2+|x|^{2}\right)^{5 / 2}} \\
a_{23}(x)=a_{32}(x)=\frac{1}{2} \frac{x_{1}^{3} \log \left(2+|x|^{2}\right)}{\left(2+|x|^{2}\right)^{5 / 2}} \\
a_{33}(x)=\frac{1}{2} \frac{x_{1}^{4} \log \left(2+|x|^{2}\right)}{\left(2+|x|^{2}\right)^{5 / 2}} .
\end{array}\right.
$$


Define

$$
\begin{aligned}
& X_{1}=\sqrt{\frac{\log \left(2+|x|^{2}\right)}{\left(2+|x|^{2}\right)^{1 / 2}}} \cdot-\frac{\partial}{\partial x_{1}} \\
& X_{2}=\sqrt{\frac{\log \left(2+|x|^{2}\right)}{\left(2+|x|^{2}\right)^{5 / 2}}} \cdot\left\{x_{1} \frac{\partial}{\partial x_{2}}+x_{1}^{2} \frac{\partial}{\partial x_{3}}\right\}
\end{aligned}
$$

and choose a first order partial differential operator $Y$ so that

$$
L=\frac{1}{2}\left\{X_{1}^{2}+X_{2}^{2}\right\}+Y
$$

It is now a simple task to verify that

$$
\operatorname{rank} \mathscr{L}\left(X_{1}, X_{2}\right)_{x}=3, \quad x \in R^{3} .
$$

Note that the rank of the coefficients' matrix $\left(a_{i j}(x)\right)_{i, j=1,2,3}$ is necessarily less than 3 , in particular if $x_{1}=0$, then it is equal to 1 .

It is also easy to see that the condition (3.1) is satisfied. Thus the associated diffusion is recurrent.

Examples 2 (Transient case). If there exists a cone $V$ as in Figure 1 such that with positive constants $\alpha>1, \beta$ and $\lambda_{2}$,

$$
E_{2}(x)\left(\text { or } \widetilde{E}_{2}(x)\right) \geqq \beta \cdot \frac{(\log |x|)^{\alpha}}{|x|^{d^{d-2}}}
$$

for all $x \in V,|x|>\lambda_{2}$, then the associated

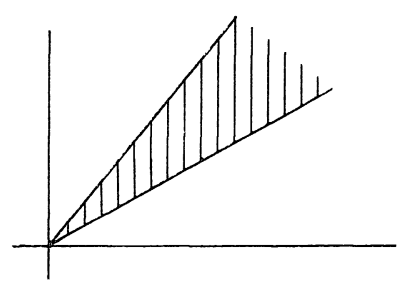

Figure 1 diffusion is transient. For the special case that

$$
L=\sum_{i=1}^{d} \frac{\partial}{\partial x_{i}}\left(a_{i}(x) \frac{\partial}{\partial x_{i}}\right)
$$

we have that if

$$
\min _{i=1, \ldots, d} a_{i}(x) \geqq \beta \cdot \frac{(\log |x|)^{\alpha}}{|x|^{d-2}}
$$

for the above $x$, the diffusion process is transient.

(i) $(d=3)$. Let $L$ be an elliptic operator defined by

$$
L=\frac{\partial^{2}}{\partial x_{1}^{2}}+x_{1}^{2} \frac{\partial^{2}}{\partial x_{2}^{2}}+x_{2}^{2} \frac{\partial^{2}}{\partial x_{3}^{2}} \text {. }
$$


Then the operator $L$ becomes a hypoelliptic one satisfying the fundamental asssumption (*) in Chapter 2. It is also easy to verify the assumption in Theorem D. Consequently the process associated with the above operator $L$ is transient.

In the next stage, we want to compare our results with those of Friedman [7] and Hasminskii [8].

We first explain briefly their main results.

Let $L$ be a strictly elliptic differential operator defined by

$$
L=\sum_{i, j=1}^{d} a_{i j} \frac{\partial^{2}}{\partial x_{i} \partial x_{j}}+\sum_{i=1}^{d} b_{i} \frac{\partial}{\partial x_{i}}
$$

where $a_{i j} \in C^{2}\left(R^{d}\right)$, symmetric and $b_{i} \in C^{1}\left(R^{d}\right)$. Denote the associated diffusion by $\left(X_{t}, P_{x}\right)$.

Define

$$
S(x)=\frac{1}{|x|}\left\{\frac{|x|^{2} \sum_{i=1}^{d}\left\{a_{i i}(x)+x_{i} b_{i}(x)\right\}}{\sum_{i, j=1}^{d} a_{i j}(x) x_{i} x_{j}}-1\right\}
$$

for $x \neq 0$ and

$$
S_{+}(r)=\max _{|x|=r} S(x), \quad S_{-}(r)=\min _{|x|=r} S(x)
$$

for $r>0$.

Constructing some semimartingales, they have shown

Theorem 3. 1. If $\int_{1}^{\infty} \exp \left[-\int_{1}^{r} S_{+}(u) d u\right] d r=+\infty$, then the process is recurrent.

Theorem 3. 2. If $\int_{1}^{\infty} \exp \left[-\int_{1}^{r} S_{-}(u) d u\right] d r<+\infty$, then the process is transient.

See Friedman [7], Chapter 9, for the proofs of the above results. In order to compare their theorems with ours, we restrict our consideration to the selfadjoint case. Therefore, from now on, it is assumed that 


$$
b_{i}(x)=\sum_{j=1}^{d} \frac{\partial a_{j i}(x)}{\partial x_{j}}, \quad i=1, \ldots, d
$$

The following theorem shows that Theorem A is a generalization of Theorem 3.1 in the selfadjoint case. that

Theorem 3. 3. If $\int_{1}^{\infty} \exp \left[-\int_{1}^{r} S_{+}(u) d u\right] d r=+\infty$, then it also holds

$$
\int_{1}^{\infty} r^{1-d} \bar{E}_{1}^{-1}(r) d r=+\infty
$$

The proof of the above theorem uses the one dimensional diffusion operator defined by

$$
\bar{L}=\frac{d}{d r}\left(r^{d-1} \bar{E}_{1}(r) \frac{d}{d r}\right), \quad r>0 .
$$

Lemma 3.1. Let $v(x)=u(|x|)$ be a smooth function in $|x|>1$. Then

$$
\left(\bar{L}_{r} u\right)(r)=\left[\int_{|x|=r}\left(L_{x} v\right)(x) d s(x)\right] /\left|S^{d-1}\right|,
$$

for $r>1$ where $d s(x)$ is the surface element of $|x|=r$.

Proof. By a simple compution, we obtain

$$
\begin{aligned}
\int_{|x|=r}(L v)(x) d s(x) & =\int_{|x|=r} E_{1}(x) d s(x) \cdot \frac{d^{2} u(r)}{d r^{2}} \\
& +\int_{|x|=r} \Gamma \cdot(A V|x|) d s(x) \cdot \frac{d u(r)}{d r} .
\end{aligned}
$$

The first term is equal to

$$
\left|S^{d-1}\right| \cdot r^{d-1} \bar{E}_{1}(r) \frac{d^{2} u(r)}{d r^{2}} .
$$

Therefore it suffices to show that

$$
\int_{|x|=r} \nabla(A \nabla|x|) d s(x)=\left|S^{d-1}\right| \cdot \frac{d}{d r}\left(r^{d-1} \bar{E}_{1}(r)\right) .
$$


Now consider, for $r>1$

$$
\begin{aligned}
& \left|S^{d-1}\right| r^{d-1} \bar{E}_{1}(r)-\left|S^{d-1}\right| \bar{E}_{1}(1) \\
& \quad=\int_{|x|=r}\left(A D|x|, n_{+}(x)\right) d s(x)+\int_{|x|=1}\left(A D|x|, n_{-}(x)\right) d s(x)
\end{aligned}
$$

where $n_{+}(x)=-n_{-}(x)=\nabla|x|$,

$$
=\int_{1<|x|<r} \nabla(A \nabla|x|) d x \text {, by the divergence theorem. }
$$

So by differentiating them with respect to $r$, one obtains

$$
\left|S^{d-1}\right| \frac{d}{d r}\left(r^{d-1} \bar{E}_{1}(r)\right)=\int_{|x|=r} \nabla(A \nabla|x|) d s(x) . \quad \text { Q. E. D. }
$$

Proof of Theorem 3.3. First define the following functions

$$
\begin{aligned}
& \psi_{\rho}(r)=\frac{\int_{r}^{\rho} u^{1-d} \bar{E}_{1}^{-1}(u) d u}{\int_{1}^{\rho} u^{1-d} \bar{E}_{1}^{-1}(u) d u} \\
& \Psi_{\rho}(r)=\frac{\int_{r}^{\rho} \exp \left[-\int_{1}^{u} S_{+}(t) d t\right] d t}{\int_{1}^{\rho} \exp \left[-\int_{1}^{u} S_{+}(t) d t\right] d t}
\end{aligned}
$$

for $r \in[1, \rho]$.

Then it is clear that

$$
\left\{\begin{array}{l}
\left(\bar{L} \phi_{\rho}\right)(r)=0 \quad \text { on } \quad(1, \rho) \\
\psi_{\rho}(1)=1, \quad \phi_{\rho}(\rho)=0 \\
\Psi_{\rho}(1)=1, \quad \Psi_{\rho}(\rho)=0
\end{array}\right.
$$

We want to show that

$$
\left(\bar{L} \Psi_{\rho}\right)(r) \geqq 0 \text { on }(1, \rho) .
$$

We now compute $L u_{\rho}(x)$, where $u_{\rho}(x)=\Psi_{\rho}(|x|)$,

$$
\begin{aligned}
\left(L u_{\rho}\right)(x) & =E_{1}(x)\left\{\frac{d^{2} \Psi_{\rho}(|x|)}{d|x|^{2}}+S(x)-\frac{d \Psi_{\rho}(|x|)}{d|x|}\right\} \\
& \geqq E_{1}(x)\left\{\frac{d^{2} \Psi_{\rho}(|x|)}{d|x|^{2}}+S_{+}(|x|) \frac{\left.d \Psi_{\rho}(\mid x)_{1}\right)}{d|x|}\right\}
\end{aligned}
$$

since $\Psi_{p}(|x|)$ is decreasing with respect to $|x|$. From the 
definition of $\Psi_{\rho}$, we see that

$$
\frac{d^{2} \Psi_{\rho}(|x|)}{d|x|^{2}}+S_{+}(|x|) \frac{d \Psi_{\rho}(|x|)}{d|x|}=0
$$

Thus we have shown $\left(L u_{\rho}\right)(x) \geqq 0$ in $1<|x|<\rho$. Combining this inequality with Lemma 3.1, (3.6) follows immediately. Using (3.5), (3.6) and the maximum principle, we can conclude that

$$
\Psi_{\rho}(r) \leqq \psi_{\rho}(r) \text { for } r \in(1, \rho) .
$$

From the assumption of Theorem 3.3, we have

$$
\lim _{\rho>+\infty} \Psi_{\rho}(r)=1 \text { for every } r>1
$$

and so $\lim _{\rho>+\infty} \psi_{\rho}(r)=1$ for $r>1$.

This implies that $\int_{1}^{\infty} r^{1-d} \bar{E}_{1}^{-1}(r) d r=+\infty$. $\quad$ Q. E D.

Finally we shall exhibit a few examples which Friedman and Hasminskii's criterions do not cover.

Examples. (i) $(d=2)$. Suppose $L$ is given by

$$
L=\left(3+\sin x_{2}\right) \frac{\partial^{2}}{\partial x_{1}^{2}}+\frac{\partial^{2}}{\partial x_{2}^{2}} .
$$

This satisfies the inequality (3.1). Consequently the associated process is recurrent. Now computing $S(x)$ for the operator, one obtains

$$
S(x)=\frac{1}{|x|} \frac{x_{2}^{2}\left(3+\sin x_{2}\right)+x_{1}^{2}}{x_{1}^{2}\left(3+\sin x_{2}\right)+X_{2}^{2}} .
$$

Taking the maximum of the function $S(x)$ on $|x|=r$, we have a lower bound,

$$
S_{+}(r)=\max _{|x|=r} S(x) \geqq \frac{2}{r}, \quad r>1 .
$$

It is now easy to see that

$$
\int_{1}^{\infty} \exp \left[-\int_{1}^{r} S_{+}(u) d u\right] d r<+\infty .
$$

This shows that Friedman and Hasminskii's test for recurrence does not cover the above case and that the converse of Theorem 3.3 is not valid. 
(ii) (d=2) Let $L$ be defined by

$$
L=a_{1}\left(x_{2}\right) \frac{\partial^{2}}{\partial x_{1}^{2}}+a_{2}\left(x_{1}\right) \frac{\partial^{2}}{\partial x_{2}^{2}}
$$

where $a_{1}, a_{2}$ are strictly positive and

$$
\left.\begin{array}{l}
a_{1}\left(x_{2}\right) \geqq \gamma \times\left(\log \left|x_{2}\right|\right)^{\alpha} \\
a_{2}\left(x_{1}\right) \geqq \delta \times\left(\log \left|x_{1}\right|\right)^{\beta}
\end{array}\right\} \quad \text { as }\left|x_{1}\right|,\left|x_{2}\right| \nearrow+\infty
$$

with positive constants $\alpha, \beta>1$ and $\gamma, \delta$.

It is easy to verify that the inequality (3.2) holds in the cone domain $\left\{x=\left(x_{1}, x_{2}\right) ; \frac{1}{2} x_{1}<x_{2}<\frac{3}{2} x_{1}\right\}$. So the associated diffusion is transient. On the other hand, we have

$$
S(x)=\frac{1}{|x|} \frac{a_{1}\left(x_{2}\right) x_{2}^{2}+a_{2}\left(x_{1}\right) x_{1}^{2}}{a_{1}\left(x_{2}\right) x_{1}^{2}+a_{2}\left(x_{1}\right) x_{2}^{2}} .
$$

If $x_{1}=x_{2}$, then $S(x)=\frac{1}{|x|}$. This implies that

$$
S_{-}(r) \leqq \frac{1}{r}
$$

which shows that the integral

$$
\int_{1}^{\infty} \exp \left[-\int_{1}^{r} S_{-}(u) d u\right] d r
$$

is divergent. Thus Theorem 3.2 is of no help in proving transience.

\section{Chapter 4. Rate of Escape}

Let $L=\sum_{i, j=1}^{d} \frac{\partial}{\partial x_{i}}\left(a_{i j} \frac{\partial}{\partial x_{j}}\right)$ be a uniformly elliptic operator i. e. assume that there exists a positive constant $\lambda>1$ such that for all $(x, \xi) \in$ $\boldsymbol{R}^{d} \times \boldsymbol{R}^{d}$

$$
\lambda^{-1}|\xi|^{2} \leqq \sum_{i, j=1}^{d} a_{i j}(x) \xi_{i} \xi_{j} \leqq \lambda|\xi|^{2}
$$

We further assume the smoothness of the coefficients $a_{i j} \in C^{\infty}\left(R^{d}\right)$ which ensures the existence of the unique fundamental solution $p_{t}(x, y)$ of $\partial / \partial t-L$. See McKean [17]. 
Let $\left(X_{t}, P_{x}\right), x \in R^{d}$ be the associated diffusion in $R^{d}$. The following theorem is an extension of the Dvoretsky-Erdös' test for the Brownian motion in space. (See Dvoretsky-Erdös [6].)

Theorem $\mathbf{E} \quad(d \geqq 3)$. If (4.1) holds and $0<g(t) \searrow$ as $t \nearrow+\infty$, then

$$
P_{x}\left[\left|x_{t}\right| \leqq \sqrt{t} g(t), i . o ., t \nearrow+\infty\right]=1 \text { or } 0
$$

according as $\int^{+\infty} g(t)^{d-2} d t / t=+\infty$ or $<+\infty$.

Our proof of this theorem is based on the following result which is essentially due to Aronson [2].

Lemma 4. 1. There exist positive constants $\lambda_{i}, M_{i}, i=1,2$ such that

$$
\frac{M_{1}}{t^{d / 2}} \exp \left[-\frac{\lambda_{1}|x-y|^{2}}{t}\right] \leqq p_{t}(x, y) \leqq \frac{M_{2}}{t^{d / 2}} \exp \left[-\frac{\lambda_{2}|x-y|^{2}}{t}\right]
$$

for every $(t, x, y) \in(0,+\infty) \times R^{d} \times R^{d}$.

Proof. The upper bound has been given by Aronson [1]. To obtain the global lower bound, we need the following Harnack inequality of Moser [18].

Harnack inequality: If $u$ is a positive solution of $\partial / \partial t-L, i . e_{.}$, $(\partial / \partial t-L) u=0$ in $(0, T) \times R^{d}$, then one has

$$
\log \frac{u(s, y)}{u(t, x)} \leqq C\left\{\frac{|x-y|^{2}}{t-s}+\log t / s+1\right\}
$$

for $0<s<t<T, x, y \in R^{d}$ where $C$ is a positive constant which depends only on $d$ and $\lambda$.

The lower bound: Step i). There exist constants $C_{i}>0, i=1,2$ such that

$$
P_{x}\left[\left|x_{t}-x\right| \leqq C_{1} \sqrt{t}\right] \geqq C_{2} \quad \text { for }(t, x) \in R^{+} \times R^{d} .
$$

This follows directly from the inequality, 


$$
E_{x}\left[\left|x_{t}-x\right|^{2}\right] \leqq C_{3} t
$$

which comes from the upper bound.

Step ii). Set $u(t, y) \equiv p_{t}(x, y)$ for a fixed $x \in R^{d}$. Applying Harnack's inequality for $\frac{\partial}{\partial t}-L$ in the domain $\left\{|y-x|^{2} \leqq C_{1}^{2} t\right\}$, we get

$$
u(t / 2, y) \leqq u(t, x) \cdot \exp \left\{2 C \cdot C_{1}^{2}+C \log 2+C\right\} .
$$

By step i), it is possible to choose a point $y_{0}(x) \in R^{d}$ such that

$$
\left|y_{0}(x)-x\right| \leqq C_{1} \sqrt{t}
$$

and

$$
u\left(t / 2, y_{0}(x)\right) \geqq M / t^{d / 2}
$$

where $M$ depends only on $C_{1}, C_{2}$ and $d$. Therefore one obtains

$$
M / t^{d / 2} \leqq u\left(t / 2, y_{0}(x)\right) \leqq u(t, x) \exp \left\{2 C \cdot C_{1}^{2}+C \log 2+C\right\} .
$$

Thus one has

$$
p_{t}(x, x) \equiv u(t, x) \geqq \tilde{M} / t^{d / 2} \text { for }(t, x) \in(0, \infty) \times R^{d}
$$

with some positive consiant $\tilde{M}$ independent of $t, x$.

Step iii). Applying Harnack's inequality once more, we obtain

$$
\frac{u(t / 2, x)}{u(t, y)} \leqq \exp \left\{\frac{2 C|x-y|^{2}}{t}+C \log 2+C\right\}
$$

(4.5) with $t$ replaced by $t / 2$ now yields,

$$
p_{t}(x, y) \equiv u(t, y) \geqq \frac{M_{1}}{t^{d / 2}} \exp \left[-\frac{\lambda_{1}|x-y|^{2}}{t}\right]
$$

for every $(t, x, y) \in(0, \infty) \times R^{d} \times R^{d}$.

Q.E.D.

From Lemma 4.1, it is easy to see that the 0-order Green function defined by

$$
G(x, y)=\int_{0}^{\infty} p_{t}(x, y) d t
$$

satisfies the inequalities,

$$
\frac{C_{4}}{|x-y|^{d-2}} \leqq G(x, y) \leqq \frac{C_{5}}{|x-y|^{d-2}}
$$


for $x, y \in R^{d},(d \geqq 3)$ and some positive constants $C_{4}, C_{5}$.

Define

$$
\begin{aligned}
& \tau_{r}^{y}=\inf \left\{t \geqq 0:\left|x_{t}-y\right| \leqq r\right\} \\
& \sigma_{\rho}^{y}=\inf \left\{t \geqq 0:\left|x_{t}-y\right| \geqq \rho\right\}
\end{aligned}
$$

for $y \in R^{d}, 0<r<\rho<+\infty$. Then

$$
u\left(x_{t \wedge \tau_{r}^{y} \wedge \Delta_{\rho}^{y}}\right) \equiv G\left(x_{t \wedge \wedge_{r}^{y} \wedge \wedge_{\rho}^{y}}, y\right)
$$

is a $P_{x}$-martingale for $r<|x-y|<\rho$. Using this, one has the following

Lemma 4. 2. For some positive constants $C_{6}, C_{7}$,

$$
C_{6}\left(\frac{r}{|x-y|}\right)^{d-2} \leqq P_{x}\left[\tau_{r}^{y}<+\infty\right] \leqq C_{7}\left(\frac{r}{|x-y|}\right)^{d-2} \text { for }|x-y|>r .
$$

Proof. Making use of the martingale defined above and the bounds for $G(x, y)$,

$$
\frac{C_{4}}{|x-y|^{d-2}} \leqq u(x)=E_{x}\left[u\left(x_{t \wedge \tau_{r}^{y} \wedge_{\rho}^{y}}\right)\right] .
$$

As $t \nearrow+\infty$, this tends to

$$
\begin{aligned}
E_{x}\left[u\left(x_{\tau_{r}^{y} \wedge \sigma_{\rho}^{y}}\right)\right] & =E_{x}\left[u\left(x_{\tau_{r}^{y}}\right), \tau_{r}^{y}<\sigma_{\rho}^{y}\right]+E_{x}\left[u\left(x_{\sigma_{\rho}^{y}}\right), \tau_{r}^{y}>\sigma_{\rho}^{y}\right] \\
& \leqq \frac{C_{5}}{r^{d-2}} \cdot P_{x}\left[\tau_{r}^{y}<\sigma_{\rho}^{y}\right]+\frac{C_{5}}{\rho^{d-2}} P_{x}\left[\tau_{r}^{y}>\sigma_{\rho}^{y}\right] .
\end{aligned}
$$

If we now let $\rho \nearrow+\infty$, we obtain

$$
\frac{C_{4}}{|x-y|^{d-2}} \leqq \frac{C_{5}}{r^{d-2}} P_{x}\left[\tau_{r}^{y}<+\infty\right], \text { for }|x-y|>r .
$$

Hence $P_{x}\left[\tau_{r}^{y}>+\infty\right] \geqq \frac{C_{4}}{C_{5}}\left(\frac{r}{|x-y|}\right)^{d-2}$. Similarly we can obtain the upper bound.

Q. E. D.

Lemma 4. 3. For $T \geqq 0, r \geqq 0$, define $Q_{y}(r, T)$ by

$$
Q_{y}(r, T)=P_{y}\left[\left|x_{t}-y\right| \leqq r \text {, for some } t \geqq T\right] \text {, }
$$

then 


$$
C_{8}\left(\frac{r}{\sqrt{T}}\right)^{d-2} \exp \left[\frac{-\lambda_{1} r^{2}}{T}\right] \leqq Q_{y}(r, T) \leqq C_{9}\left(\frac{r}{\sqrt{T}}\right)^{d-2}
$$

where $C_{8}, C_{9}$ are positive constants.

Proof. Using the Markov property and Lemma 4.2, we obtain

$$
\begin{gathered}
\int_{|x-y| \geqq r} C_{6}\left(\frac{r}{|x-y|}\right)^{d-2} p_{T}(y, x) d x \leqq Q_{y}(r, T) \\
\leqq \int_{R^{d}} C_{7}\left(\frac{r}{|x-y|}\right)^{d-2} p_{T}(y, x) d x
\end{gathered}
$$

and so

$$
\begin{aligned}
Q_{y}(r, T) & \leqq \int_{R^{d}} C_{7} M_{2}\left(\frac{r}{\sqrt{T}}\right)^{d-2} \frac{1}{|x-y|^{d-2}} \exp \left[-\lambda_{2}|x-y|^{2}\right] d x \\
& =C_{9}\left(\frac{r}{\sqrt{T}}\right)^{d-2} .
\end{aligned}
$$

Similarly we can obtain the lower bound,

$$
Q_{y}(r, T) \geqq C_{8}\left(\frac{r}{\sqrt{T}}\right)^{d-2} \exp \left[\frac{-\lambda_{1} r^{2}}{T}\right] . \quad \text { Q. E. D. }
$$

Lemma 4. 4. Let $K>1$ be such that

$$
C_{10} \equiv C_{8} \exp \left(-\lambda_{1}\right)-C_{9}\left(\frac{1}{\sqrt{K}}\right)^{d-2}>0 .
$$

Define

(4. 10) $\quad P_{y}(r, T, K) \equiv P_{y}\left\{\left|x_{t}-y\right| \leqq r\right.$, for some $\left.t \in[T, K T]\right\}$.

Then for $\sqrt{T} \geqq r>0$

$$
P_{y}(r, T, K) \geqq C_{10}\left(\frac{r}{\sqrt{T}}\right)^{d-2} .
$$

Proof. From the definition of $P_{y}(r, T, K)$ and $Q_{y}(r, T)$,

$$
P_{y}(r, T, K) \geqq Q_{y}(r, T)-Q_{y}(r, K T) .
$$

Applying Lemma 4.3, we obtain

$$
P_{y}(r, T, K) \geqq C_{8}\left(\frac{r}{\sqrt{T}}\right)^{d-2} \exp \left(\frac{-\lambda_{1} r^{2}}{T}\right)-C_{9}\left(\frac{r}{\sqrt{K T}}\right)^{d-2}
$$




$$
\geqq\left\{C_{8} \exp \left(-\lambda_{1}\right)-C_{9}\left(\frac{1}{\sqrt{K}}\right)^{d-2}\right\}\left(\frac{r}{\sqrt{T}}\right)^{d-2} \cdot \quad \text { Q. E. D. }
$$

We are now in a position to prove our theorem.

Proof of Theorem E. We first note that it is enough to consider the case,

$$
1 \geqq g(t) \searrow 0 \text { as } t \nearrow+\infty \text {. }
$$

Now define

$$
\begin{aligned}
& E_{k}=\left\{\omega:\left|x_{t}(\omega)\right| \leqq \sqrt{t} g(t) \text { for some } t \in\left[K^{k}, K^{k+1}\right]\right\} \\
& \bar{E}_{k}=\left\{\omega:\left|x_{t}(\omega)\right| \leqq \sqrt{K^{k+1}} g\left(K^{k}\right) \text { for some } t \in\left[K^{k}, K^{k+1}\right]\right\}
\end{aligned}
$$

for every positive integer $k$. Since $g$ is decreasing, $E_{k} \subset \bar{E}_{k}$ for $k \geqq 1$. By Lemma 4.3,

$$
P_{0}\left(\bar{E}_{k}\right) \leqq C_{9}\left(\frac{g\left(K^{k}\right) \sqrt{K^{k+1}}}{\sqrt{K^{k}}}\right)^{d-2}=C_{9} \cdot K^{d-2 / 2} \cdot\left\{g\left(K^{k}\right)\right\}^{d-2}
$$

We now assume the convergence of the integral in our theorem. Then

$$
\sum_{k=1}^{\infty} P_{0}\left(\bar{E}_{k}\right) \leqq O(1) \sum_{k=1}^{\infty}\left\{g\left(K^{k}\right)\right\}^{d-2}=O(1) \int^{+\infty} g(t)^{d-2} \frac{d t}{t}<+\infty
$$

and so by the Borel-Cantelli Lemma, the left hand side of (4.2) equals zero.

Next we consider the divergent case. Using a result of Kochen and Stone [13], we shall first show that

$$
P_{0}\left[\left|x_{t}\right| \leqq \sqrt{t} g(t), \text { i.o., } t \nearrow+\infty\right]=1
$$

under the additional assumption

$$
\frac{1}{(\log t)^{2}} \leqq g(t)^{d-2} \searrow 0 \text { as } t \nearrow+\infty .
$$

In order to do this, we introduce the following definition

$$
E_{k, y}^{\lambda}=\left\{\omega:\left|x_{t}(\omega)-y\right| \leqq \lambda \sqrt{K^{k}} g\left(K^{k+1}\right) \text { for some } t \in\left[K^{k}, K^{k+1}\right]\right\}
$$

for every $y \in R^{d}$, positive integer $k$ and positive $\lambda \leqq 1$. From Lemma 
4. 4, it follows that

$$
P_{y}\left(E_{k, y}^{2}\right) \geqq C_{10} \cdot \lambda^{d-2}\left\{g\left(K^{k+1}\right)\right\}^{d-2}
$$

and so

$$
\sum_{k=1}^{\infty} P_{y}\left(\underline{E}_{k, y}^{\lambda}\right) \geqq C_{11} \lambda^{d-2} \int^{+\infty} g(t)^{d-2} \frac{d t}{t}=+\infty
$$

Define a stopping time by

$$
\sigma_{j, y}^{2}(\omega)=\left\{\begin{array}{l}
\inf \left\{t \geqq 0 ;\left|x_{t}(\omega)-y\right| \leqq \lambda \sqrt{K^{j}} g\left(K^{j+1}\right), K^{j} \leqq t \leqq K^{j+1}\right\} \\
+\infty, \text { if no such } t \text { exists. }
\end{array}\right.
$$

Using the strong Markov property, for a pair $i>j$,

$$
\begin{gathered}
P_{y}\left\{E_{i, y}^{\lambda} \cap \underline{E}_{j, y}^{\lambda}\right\}=\int_{K^{j}}^{K^{j+1}} P_{y}\left\{\left|x_{t}-y\right| \leqq \lambda \sqrt{K^{i}} g\left(K^{i+1}\right)\right. \text { for some } \\
\left.t \in\left[K^{i}, K^{i+1}\right] \mid \sigma_{j, y}^{\lambda}=s\right\} \quad P_{y}\left\{\sigma_{j, y}^{\lambda} \in d s\right\} \\
\leqq \max _{\left\{|z-y| \leqq \lambda^{2} \bar{K}^{j}\left(K^{j+1}\right)\right\}} P_{z}\left\{\left|x_{t}-z\right| \leqq \lambda g\left(K^{i+1}\right) \sqrt{K^{i}}+\lambda g\left(K^{j+1}\right) \sqrt{K^{j}},\right. \\
\text { for some } \left.t \geqq K_{i}-K^{j+1}\right\} P_{y}\left\{\sigma_{j, y}^{\lambda} \leqq K^{j+1}\right\} .
\end{gathered}
$$

By Lemma 4.3, we have that

$$
\begin{aligned}
P_{z}\left\{\left|x_{t}-z\right|\right. & \left.\leqq \lambda \sqrt{K^{i}} g\left(K^{i+1}\right)+\lambda \sqrt{K^{j}} g\left(K^{j+1}\right) \text { for some } t \geqq K^{i}-K^{j+1}\right\} \\
& \leqq C_{9}\left\{\frac{\lambda g\left(K^{i+1}\right) \sqrt{K^{i}}+\lambda g\left(K^{j+1}\right) \sqrt{K^{j}}}{\sqrt{K^{i}-K^{j+1}}}\right\}^{d-2} \\
& =C_{9} \cdot \lambda^{d-2}\left\{\frac{g\left(K^{i+1}\right) \sqrt{K^{i}}+g\left(K^{j+1}\right) \sqrt{K^{j}}}{\sqrt{K^{i}-K^{j+1}}}\right\}^{d-2} \cdot
\end{aligned}
$$

On the other hand, (4.13) implies that

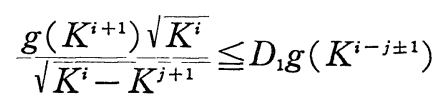

and

$$
\frac{g\left(K^{j+1}\right) \sqrt{K^{j}}}{\sqrt{K^{i}-K^{j+1}}} \leqq \frac{D_{2}}{\left(\log \frac{K^{i}-\bar{j}+\overline{1}}{1^{2 / d-2}}\right.} \leqq D_{2} g\left(K^{i-j+1}\right)
$$

for $i \geqq j+2$, where $D_{1}$ and $D_{2}$ are positive constants which are independent of $i, j, \lambda$ and $g$ above. This combined with the inequality 
(4. 14) shows that for $i \geqq j+2$

$$
P_{y}\left\{E_{i, y}^{\lambda} \cap \underline{E}_{j, y}^{\lambda}\right\} \leqq C_{12} P_{y}\left\{\underline{E}_{i-j, y}^{\lambda}\right\} \cdot P_{y}\left\{\underline{E}_{j, y}^{\lambda}\right\}
$$

with a positive constant $C_{12}$ independent of $\lambda, y$ and $g$. For $i=j+1$, it is obvious that

$$
P_{y}\left\{\underline{E}_{i, y}^{\lambda} \cap \underline{E}_{j, y}^{\lambda}\right\} \leqq P_{y}\left(\underline{E}_{j, y}^{\lambda}\right) .
$$

So from the inequality (4.14) with $k=1$, we have that if $1 \geqq \lambda>1 / 2$,

$$
P_{y}\left\{E_{i, y}^{\lambda} \cap \underline{E}_{j, y}^{\lambda}\right\} \leqq C_{13} P_{y}\left\{\underline{E}_{i-j, y}^{\lambda}\right\} \cdot P_{y}\left\{E_{j, y}^{\lambda}\right\}
$$

for $i>j$ where $C_{13}$ is a positive constant which does not depend upon $y$ and $g$.

It is now easily seen that

$$
\varlimsup_{n>+\infty} \frac{\left\{\sum_{i=1}^{n} P_{y}\left(E_{i, y}^{2}\right)\right\}^{2}}{\sum_{j, k=1}^{n} P_{y}\left\{\underline{E}_{j, y}^{2} \cap \underline{E}_{k, y}^{2}\right\}} \geqq \frac{1}{2 C_{13}}>0
$$

for every $\lambda, y$ and $g$ above. Combining this with a result of Kochen and Stone [13], it follows that

$$
P_{y}\left\{\varlimsup_{n>+\infty} E_{n, y}^{2}\right\} \geqq \frac{1}{2 C_{13}} .
$$

This implies that

$$
P_{y}\left\{\left|x_{t}-y\right| \leqq \lambda \sqrt{t} g(t), \text { i.o., } t \nearrow+\infty\right\} \geqq \frac{1}{2 C_{13}}
$$

for every $\lambda \in\left(\frac{1}{2}, 1, y \in R^{d}\right.$ and $g$ satisfying (4.13). We want to show that

$$
P_{y}\left\{\left|x_{t}\right| \leqq \sqrt{t+u} g(t+u), \text { i.o., } t \nearrow+\infty\right\} \geqq \frac{1}{2 C_{13}}
$$

for any fixed positive $u$. First note that (4.19) gives

$$
P_{y}\left\{\left|x_{t}\right| \leqq \lambda \sqrt{t} g(t), i . o ., t \nearrow+\infty\right\} \geqq \frac{1}{2 C_{13}}
$$

because $\lim _{t>+\infty} \frac{|y|}{\sqrt{t}} \frac{y \mid}{g(t)}=0$. In order to deduce (4.20), we observe that

$$
\sqrt{t+u} g(t+u) \geqq \sqrt{t} g(t+u)
$$


and

$$
(2 g(t+u))^{d-2} \geqq \frac{2^{d-2}}{(\log (t+u))^{2}}>\frac{1}{(\log l)^{2}}
$$

for all sufficiently large $t$, where $u$ is a fixed positive number. Hence combining this with (4.21), we obtain the inequality (4.20).

Using (4.20), we shall prove

$$
P_{x}\left[\left|x_{t}\right| \leqq \sqrt{t} g(t), i . o ., t \nearrow+\infty\right]=1, x \in R^{d} .
$$

Define

$$
Y_{t}=P_{x}\left\{\left|x_{t}(\omega)\right| \leqq \sqrt{t} g(t), \text { i.o., } t \nearrow+\infty \mid \mathscr{F}_{0}^{t}\right\}
$$

where $\mathscr{F}_{0}^{t}=\sigma\left\{x_{s}, s \leqq t\right\}$. Then $Y_{t}$ is an $\mathscr{F}_{0}^{t}$-martingale. Since $Y_{t}$ is bounded, by the martingale convergence theorem we have

$$
\lim _{t \lambda+\infty} Y_{t}(\omega)= \begin{cases}1 & \text { if } \mid x_{t}(\omega) i \leqq \sqrt{t} g(t), i . o ., t \nearrow+\infty \\ 0 & \text { otherwise. }\end{cases}
$$

On the other hand, from the definition of $Y_{t}$ and the inequality (4. 20),

$$
Y_{t}(\omega)=P_{x_{t}}\left\{\left|x_{s}(\omega)\right| \leqq \sqrt{t+s} g(t+s), \text { i.o., } s \nearrow+\infty\right\} \geqq \frac{1}{2 C_{13}}>0 .
$$

This implies that $\lim _{t>+\infty} Y_{t}(\omega)=1$ a. e., i. e.,

$$
P_{x}\left[\left|x_{t}\right| \leqq \sqrt{t} g(t), i . o ., \text { as } t \nearrow+\infty\right]=1, \quad x \in R^{d} \text {. }
$$

For a general $g(t)$, define

$$
g_{1}(t)=\max \left\{g(t), \sqrt[d-2]{\frac{1}{(\log t)^{2}}}\right\} .
$$

It is evident that $g_{1}(t)$ satisfies the condition (4.13).

Therefore, from what we have shown above, it follows that

$$
P_{x}\left[\left|x_{t}\right| \leqq \sqrt{t} g_{1}(t), \text { i.o., } t \nearrow+\infty\right]=1 .
$$

On the other hand, from the convergent case,

$$
P_{x}\left[\left|x_{t}\right| \leqq \sqrt{t} \cdot \sqrt[d-2]{\frac{1}{(\log t)^{2}}}, \text { i.o., } t \nearrow+\infty\right]=1
$$

because of $\int^{+\infty} \frac{d t}{t(\log t)^{2}}<+\infty$. Hence we finally obtain that 


$$
P_{x}\left[\left|x_{t}\right| \leqq \sqrt{t} g(t), \text { i.o., } \nearrow+\infty\right]=1 \text {. }
$$

Q. E. D.

\section{Bibliography}

[1] Agmon, S., The $L^{2}$-approach to the Dirichlet problem, Ann. Scuola. Norm. Sup. Pisa, 13 (1959), 405-448.

[2] Aronson, D. G., Bounds for the fundamental solution of a parabolic equation, Bull. Amer. Math. Soc., 73 (1967), 890-896.

[3] Bony, J. M., Principe du maximum, unicité du problème de Cauchy et inegalite de Harnack des operateurs elliptiques degeneres, Ann. Inst. Fou rier, 19 (1969), 277-304.

[4] Brown, L. D., Admissible estimators, recurrent diffusions and insoluble boundary value problems, Ann. Math. Statist., 42 (1971), 855-903.

[5] Courrége, P. and Priouret, P., Recollements de processus de Markov continus, C. R. Acad. Sc. Paris, 259 (1964), 4220-4222.

[6] Dvoretsky, A. and Erdös, P., Some problems on random walks in space, Proc. Second Berkeley Sump. Math. Statist. Prob. (1951), 353-367.

[7] Friedman, A., Stochastic differential equations and applications, vol. 1 (1975), Academic Press.

[8] Hasmininskii, R. Z., Ergodic properties of recurrent diffusion processes and stabilization of the problem to the Cauchy problem for parabolic equations, T. V. 5 (1960), 196-214.

[9] Hörmander, L., Hypoelliptic second order differential equations, Acta Math., 119 (1967), 147-171.

[10] Hunt, G. A., On positive Green functions, Proc. Nat. Acad. Sci., 40 (1954), 816-818.

[11] Ichihara, K. and Kunita, H., A classification of the second order degenerate elliptic operators and its probabilistic characterization, Z. Wahr. Verw. Gebiete, 30 (1974), 235-254.

[12] Kanda, M., Regular points and Green functions in Markov processes, Jour. Math. Sci. Japan, 19 (1967), 246-269.

[13] Kochen, S. and Stone, C., A note on the Borel-Cantelli Lemma, Illinois Jour. Math. 8 (1974), 248-251.

[14] Kunita, H., General boundary conditions for multidimensional diffusion processes, Jou r. Math. Kyoto Univ., 10, (1970), 273-335.

[15] Lions, J. L. and Magenes, E., Problìme aux limites non homogènes et applications, vol. 1, Dunod Paris 1968.

[16] Littman, W., Stampacchia, G. and Weinberger, H. F., Regular points for elliptic equations with discontinuous coefficients, Ann. Scuola. Norm. Sup. Pisa, 17 (1963), 43-76.

[17] McKean, H. P., Stochastic integ rals, New York Academic Press (1969).

[18] Moser, J., A Harnack inequality for parabolic differential equations, Comm. Pu re Appl. Math., 17 (1964), 101-134.

[19] Stampacchia, G., Le problème de Dirichlet pour les equations elliptiques du second ordre a coefficients discontinus, Ann. Inst. Fourier, 15 (1965), 189-258.

[20] Stroock, D. W. and Varadhan, S. R. S., On the support of diffusion processes with applications to the strong maximum principle, Proc. Sixth Berkeley Symp. Math. Statist. Prob.

[21] Stroock, D. W. and Varadhan, S. R. S., On degenerate elliptic-parabolic operators of second order and their associated diffusions, Comm. Pure Appl. Math., 25 (1972), 651 $-713$.

[22] Yosida, K., Functional Analysis, Springer-Verlag (1966). 